\title{
Magnetosheath waves under very low solar wind dynamic pressure: Wind/Geotail observations
}

\author{
C. J. Farrugia ${ }^{1}$, F. T. Gratton ${ }^{2}$, G. Gnavi ${ }^{2}$, H. Matsui ${ }^{1}$, R. B. Torbert ${ }^{1}$, D. H. Fairfield ${ }^{3}$, K. W. Ogilvie $^{3}$, R. P. Lepping ${ }^{3}$, \\ T. Terasawa ${ }^{4}$, and T. Mukai, Y. Saito ${ }^{5}$ \\ ${ }^{1}$ Space Science Center and Department of Physics, University of New Hampshire, NH, USA \\ ${ }^{2}$ Instituto de Física del Plasma, CONICET and FCEyN, University of Buenos Aires, Buenos Aires, Argentina \\ ${ }^{3}$ NASA Goddard Space Flight Center, Greenbelt, MD, USA \\ ${ }^{4}$ Department of Earth and Planetary Physics, University of Tokyo, Tokyo, Japan \\ ${ }^{5}$ Institute of Space and Astronautical Sciences, Kanagawa, Japan
}

Received: 2 October 2004 - Revised: 11 February 2005 - Accepted: 24 February 2005 - Published: 3 June 2005

\begin{abstract}
The expanded bow shock on and around "the day the solar wind almost disappeared" (11 May 1999) allowed the Geotail spacecraft to make a practically uninterrupted 54h-long magnetosheath pass near dusk (16:30-21:11 magnetic local time) at a radial distance of 24 to $30 R_{E}$ (Earth radii). During most of this period, interplanetary parameters varied gradually and in such a way as to give rise to two extreme magnetosheath structures, one dominated by magnetohydrodynamic (MHD) effects and the other by gas dynamic effects. We focus attention on unusual features of electromagnetic ion wave activity in the former magnetosheath state, and compare these features with those in the latter. Magnetic fluctuations in the gas dynamic magnetosheath were dominated by compressional mirror mode waves, and leftand right-hand polarized electromagnetic ion cyclotron (EIC) waves transverse to the background field. In contrast, the MHD magnetosheath, lasting for over one day, was devoid of mirror oscillations and permeated instead by EIC waves of weak intensity. The weak wave intensity is related to the prevailing low solar wind dynamic pressures. Left-hand polarized EIC waves were replaced by bursts of right-hand polarized waves, which remained for many hours the only ion wave activity present. This activity occurred when the magnetosheath proton temperature anisotropy $\left(=T_{p, \perp} / T_{p, \|}-1\right)$ became negative. This was because the weakened bow shock exposed the magnetosheath directly to the (negative) temperature anisotropy of the solar wind. Unlike the normal case studied in the literature, these right-hand waves were not by-products of left-hand polarized waves but derived their energy source directly from the magnetosheath temperature anisotropy. Brief entries into the low latitude boundary layer (LLBL) and duskside magnetosphere occurred under such inflated conditions that the magnetospheric magnetic pressure was insufficient to maintain pressure balance. In these
\end{abstract}

Correspondence to: C. J. Farrugia

(charlie.farrugia@unh.edu) crossings, the inner edge of the LLBL was flowing sunward. The study extends our knowledge of magnetosheath ion wave properties to the very low solar wind dynamic pressure regime.

Keywords. Ionosphere (Wave-particle interactions) - Magnetospheric physics (Magnetosheath) - Radio science (Waves in plasma)

\section{Introduction}

There are two major approaches to modeling the flow of the shocked solar wind around the terrestrial magnetosphere. In the traditional approach, known as the convected gasdynamic model (CGDM) and associated with the names of Spreiter and coworkers (Spreiter et al., 1966; Spreiter and Alksne, 1969; Spreiter and Stahara, 1980), the solution for the flow around a blunt body is obtained first, neglecting the magnetic forces in the momentum equation. After that, the magnetic field is derived by passive convection in the gas dynamic flow field, using the frozen-in field condition. Although this kinematic approach decouples the solution of the flow from that of the field, it has been widely successful in explaining the gross features of the magnetosheath of magnetized planets. It becomes increasingly reliable as the Alfven Mach number of the solar wind $\left(M_{A}\right)$ increases because in the momentum equation the $\mathbf{j} \times \mathbf{B}$ force scales as $M_{A}^{-2}$ (Spreiter et al., 1966). Quantity $M_{A}\left(M_{A}^{2} \equiv V_{p}^{2} / V_{A}^{2}=\rho V_{p}^{2} /\left(B^{2} / \mu_{0}\right)\right.$, where $V_{A}$ is the Alfven speed and $\rho$ is the mass density) is related to the solar wind dynamic pressure $P_{d y n}$ by $B^{2} M_{A}^{2}=\mu_{0} P_{d y n}$, so that for constant $B$, a low $M_{A}$ implies a low $P_{d y n}$, and vice versa. However, close to the magnetopause itself, and in the absence of magnetopause reconnection at low latitudes, which can convert magnetic energy into plasma energy, the magnetic field starts to pile up at the frontside of the magnetopause, vitiating the assumptions of the CGDM. As a result, 

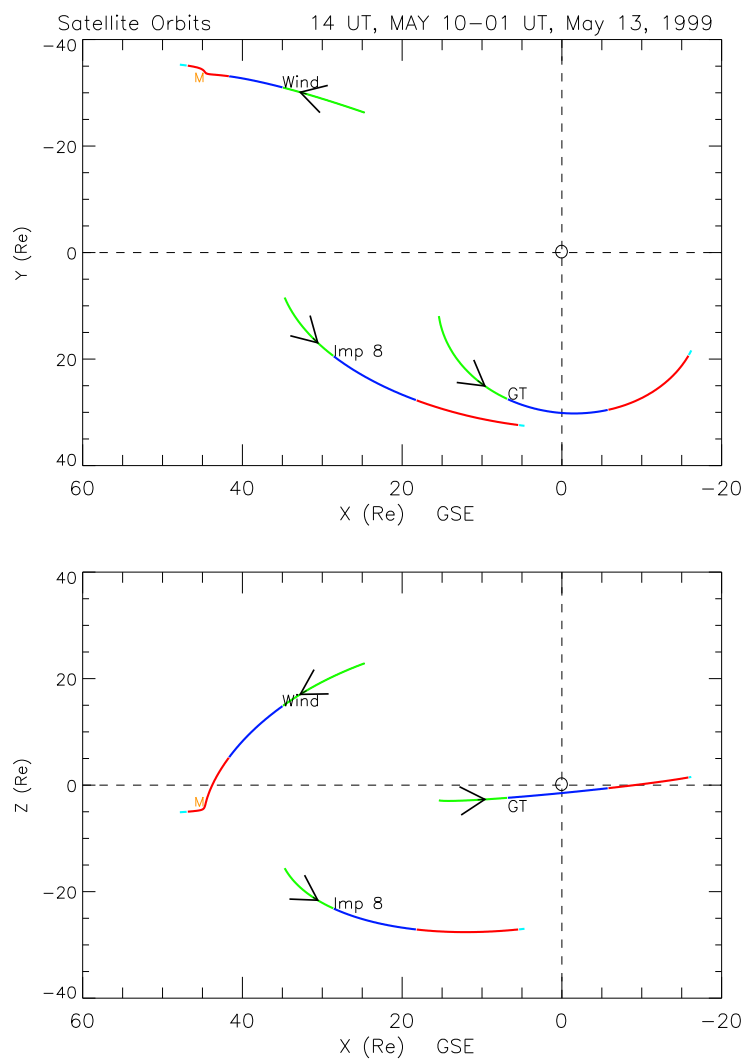

Fig. 1. The orbits of three near-Earth spacecraft data from which are discussed in this study. The top and bottom panels show, respectively, GSE XY and XZ projections. The interval plotted is 14:00 UT (10), 01:00 UT (13), 3 h longer than Geotail's magnetosheath passage near dusk, which started at 16:40 UT (10). The different colors correspond to different days.

a region of strong field and low density forms adjacent to the sunward side of the magnetopause, called the plasma depletion layer (PDL). Its thickness is proportional to $1 / M_{A}^{2}$ (Farrugia et al., 1995)

In the second approach, the influence of the interplanetary magnetic field (IMF) on the magnetosheath flow is included from the start (see, for instance, Midgley and Davis, 1963; Lees, 1964; Zwan and Wolf, 1976; Erkaev, 1988; Wang et al., 2003). In this treatment the PDL arises naturally and defines a MHD-dominated region whose flow and wave properties are different from those in the rest of the magnetosheath. On the dayside the flow is of the stagnation line type (Sonnerup, 1974; Phan et al., 1994). As a result, at the magnetopause the flow tends to align itself perpendicular to the local magnetic field. As the magnetopause is approached, the temperature anisotropy of the protons, $A_{p} \equiv T_{p, \perp} / T_{p, \|}-1$ (where the symbols " $\perp$ " and " $\|$ " are defined with respect to the background magnetic field direction) increases. Further, $A_{p}$ is found to anticorrelate with $\beta_{p, \|}$, as predicted by theory (Gary and Lee, 1994; Gary et al., 1994) and confirmed experimentally ( Anderson and Fuselier, 1993; Anderson et al., 1991, 1994).
Whereas the main body of the magnetosheath is high beta and the condition to de-stabilize the mirror mode is generally marginally satisfied (Phan et al., 1994; Hill et al., 1995), in the low $\beta$-high, positive $A_{p}$ PDL the mirror mode is stable, and instead left-hand polarized electromagnetic ion cyclotron waves (EICWs) transverse to the field are excited, as first reported by Fairfield (1976). Right-hand polarized EICW power is lower than the left-hand power and is thought to be generated as a secondary emission ("daughter" waves) from the left-hand waves. The PDL may thus be characterized by a special type of wave activity, the EIC waves. One main objective of this paper is to show that under very low $P_{d y n}$ this PDL-type wave activity extends to the main body of the magnetosheath (as judged by the distance of the spacecraft from a model magnetopause surface). Another is to show that the right-hand polarized EICWs are directly generated by the negative temperature anisotropy (and not as secondary waves) at a time when the bow shock is weak.

In an experimental work, we shall analyze ion wave activity in the magnetosheath under low $P_{d y n}$ conditions (at nearly constant $B$ ), which occurred over the extended period 10-11 May 1999. This is a much studied event, and 11 May 1999, has been dubbed "the day the solar wind almost disappeared" because solar wind densities decreased to low values of order $0.2 \mathrm{~cm}^{-3}$ and $P_{d y n}$ to $\sim 0.10 \mathrm{nPa}$, see special editions of Geophys. Res. Lett. (2000) and J. Geophys. Res. (2000). In particular, the bow shock was displaced to its most sunward position on record, about $50 R_{E}$ upstream of Earth (Fairfield et al., 2001). Because of the dilated magnetosphere and bow shock, the spacecraft Geotail spent an uninterrupted stretch of $54 \mathrm{~h}$ in the magnetosheath. There it observed many effects due to the strong influence of the IMF on the magnetosheath, which may be isolated and studied by comparing them with a long segment of the same pass when MHD effects were much attentuated. An important feature is that interplanetary parameters change very slowly during the long-duration density decrease, so that the magnetosheath traverses essentially a sequence of quasi-steady states.

The layout of the paper is as follows. After a discussion of interplanetary conditions recorded by Wind and IMP 8, we discuss in turn the magnetic field, plasma, and wave observations made by Geotail in the magnetosheath. Wave theory results are discussed in conjunction with the observed electromagnetic ion wave spectra. We then discuss the relevance of these findings to our knowledge of the magnetosheath.

\section{Wind and IMP 8 observations}

\subsection{Spacecraft orbits}

Figure 1 shows the positions of the near-Earth spacecraft Wind, IMP 8 and Geotail in a GSE XY (top panel) and $\mathrm{XZ}$ (bottom panel) projection for the period corresponding to Geotail's magnetosheath passage (14:00 UT, 10 May, 01:00 UT, 13 May). Different days are marked by different colors. The kink on Wind's orbit on 12 May occurs when 


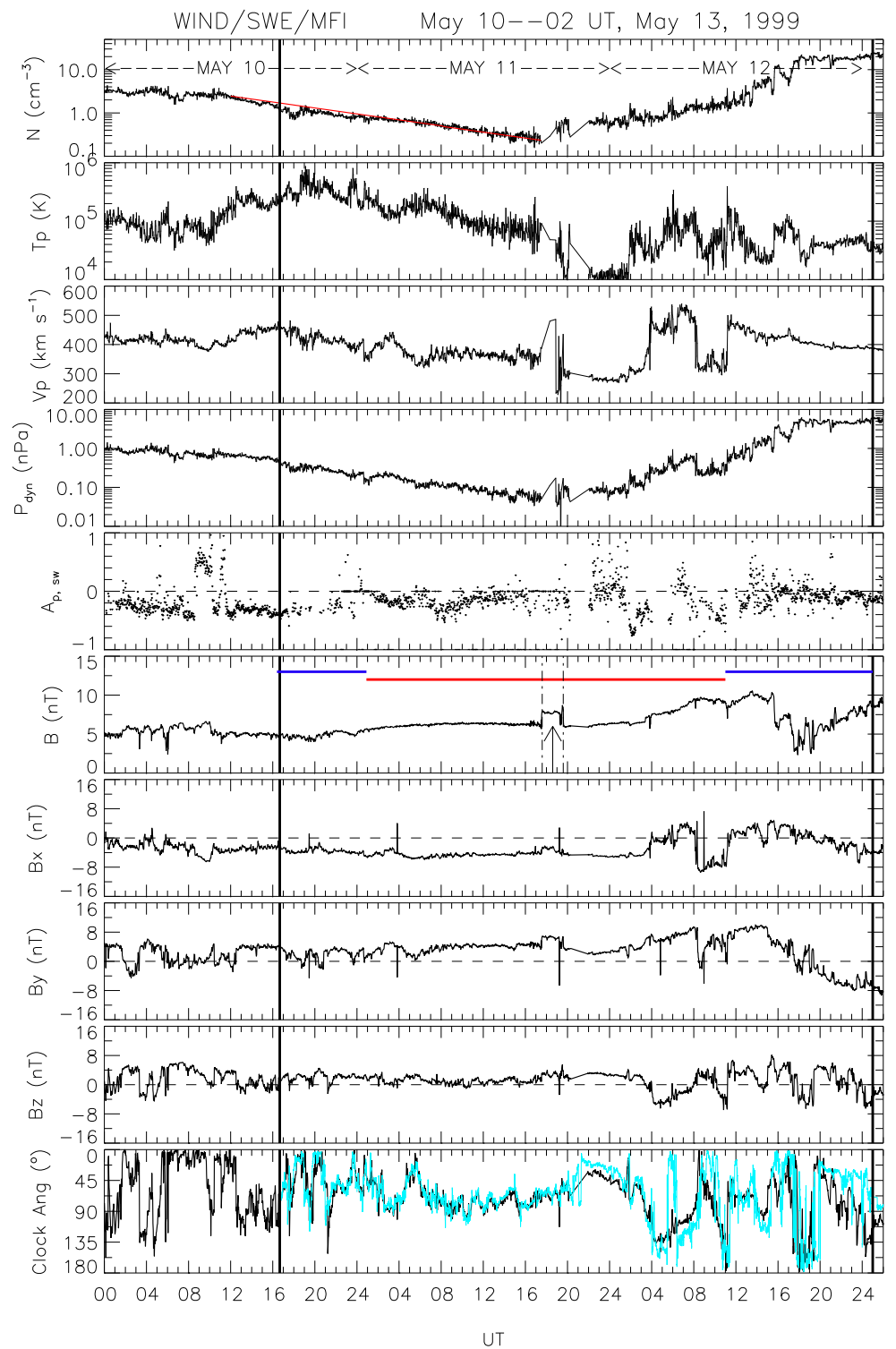

Fig. 2. Plasma and magnetic field data from the SWE and MFI investigations on Wind. GSM coordinates are used. The time resolution of the data sets are $\sim 90 \mathrm{~s}$ (plasma) and $3 \mathrm{~s}$ (magnetic field). The vertical lines bracket the interval when Geotail was in the magnetosheath. The arrow in the 5th panel marks the time when the bow shock passed over Wind. For details about the panels, see text. The light blue trace in the bottom panel is an overlay of the clock angle measured by Geotail in the magnetosheath when no lag is assumed between Wind and Geotail measurements.

the spacecraft was executing a swing-by manoeuvre near the moon (symbol "M") on 12 May.

Geotail orbits at a radial distance which varies between 24.2 to $30.3 R_{E}$ (Earth radii), and covers a magnetic local time (MLT) range from 16:25 to 21:10 MLT, with the spacecraft staying close to the ecliptic plane. Wind was on the opposite side of the Sun-Earth line to Geotail and traveling sunward. The inter-spacecraft separation orthogonal to the Sun-Earth line lies in the range 46 to $64 R_{E}$. IMP 8 is more favorably located, but no plasma data are available from this spacecraft. After introducing the Wind observations in Figs. 2 and 3, we shall cross-correlate Wind and IMP 8 measurements to ascertain that Wind data are appropriate for this study, since a distance of $\sim 60 R_{E}$ perpendicular to the SunEarth line is comparable to typical correlationlengths of the IMF in this direction (Richardson and Paulerena, 2001; Matsui et al., 2002).

Proton plasma and magnetic field data from the Solar Wind Experiment (SWE; Ogilvie et al., 1995) and the Magnetic Field Investigation (MFI; Lepping et al., 1995) on the Wind spacecraft are shown in Fig. 2. From top to bottom the panels display the proton plasma density, temperature and bulk speed, the dynamic pressure $\left(P_{d y n}\right)$, the solar wind proton temperature anisotropy, $A_{p, s w}$, the total field and its GSM components, and the IMF clock angle (i.e. the polar angle in the GSM YZ plane). The period plotted is 00:00 UT, 

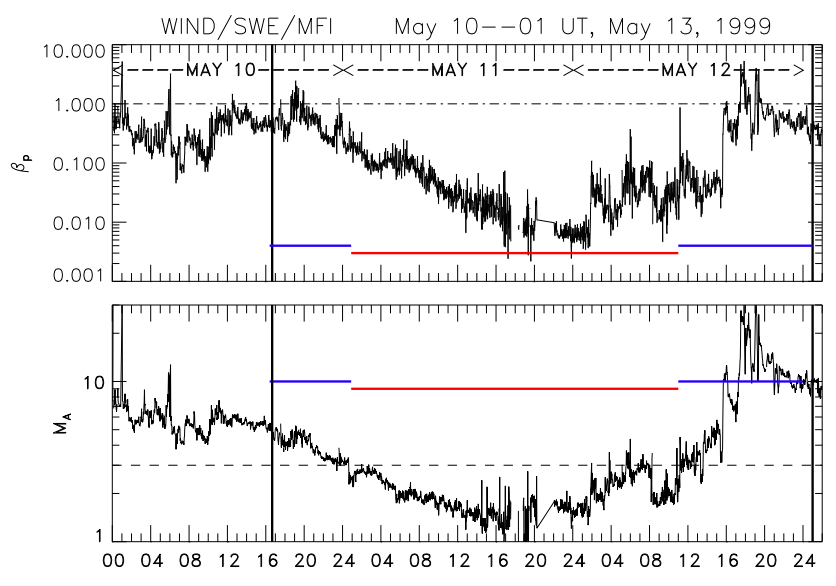

UT

Fig. 3. The proton plasma beta and the Alfven Mach number derived from Wind measurements. The vertical guidelines bracket the duration of Geotail's magnetosheath interval. Two regimes in $\beta_{p}-M_{A}$ space are indicated by the two colored horizontal bars: MHD-dominated in red; gas dynamic-dominated in blue.

10 May, 02:00 UT, 13 May 1999. The temporal resolutions of the data are $90 \mathrm{~s}$ for the plasma and $3 \mathrm{~s}$ for the magnetic field. The $A_{p, s w}$ data are 3-min averages. The vertical lines delimit the time interval when Geotail was inside the magnetosheath. (The colored horizontal bars in the $B$ panel and the light blue trace in the clock angle panel are explained below.) Aside from a brief interval 17:40-19:40 UT (11), indicated by an arrow in panel 6, when the sunward-expanding bow shock crossed Wind, this spacecraft was positioned upstream of the bow shock, being at $(32.0,-29.8,17.8) R_{E}$ and (47.7, $-35.3,-5.1) R_{E}$ (GSE coordinates) at 16:00 UT, 10 May and 01:00 UT, 13 May, respectively. (For convenience, we shall use below the notation x UT (y) to denote x UT on May y, 1999.)

At Wind, the density started its steady decrease from values of $\sim 3 \mathrm{~cm}^{-3}$ at 11:00 UT (10), reaching lowest values of $\sim 0.2 \mathrm{~cm}^{-3}$ when the bow shock passed over the spacecraft at $\sim 18: 00$ UT (11). The progressively more tenuous wind is also progressively colder and slower. In contrast, the total field is relatively steady $(<B>=5.60 \pm 0.68 \mathrm{nT})$ and is characterized by a negative $B_{x}$, a positive $B_{y}$ and has a slightly northward orientation on average. Quantity $P_{d y n}$ reaches lowest values of $\sim 0.07 \mathrm{nPa}$. The temperature anisotropy, $A_{p, s w}$ is generally negative with intermittent positive values.

The solar wind density decreases as $n_{s w}=2.45 \mathrm{e}^{-0.08 T}$ ( $\mathrm{T}$ in hours), shown by the red line in the first panel of Fig. 2 . This almost linear descent amounts to a steady decrease of $\sim 0.1 \mathrm{~cm}^{-3} \mathrm{~h}^{-1}$ in $n_{p}$ (and $0.02 \mathrm{nPa} \mathrm{h}^{-1}$ in $P_{d y n}$ ), slow enough for conditions in the magnetosheath to be considered as changing in a quasi-steady fashion.

For the same interval as Fig. 2, Fig. 3 shows the plasma beta $\beta_{p}$ and the Alfven Mach number $M_{A}$ derived from the Wind measurements. During the density decrease, the plasma beta drops by about two orders of magnitude, from values of $\sim 1$ to $\sim 0.01$, returning to typical solar wind values of $\sim 1$ in the last $10 \mathrm{~h}$ of the interval. Parameter $M_{A}$, which starts from $\sim 7$, reaches lowest values of $\sim 1.3$, only to return to values $\geq 10$ in the last $10 \mathrm{~h}$. Thus there is a $\sim 1.5$-day period, marked by the horizontal red bar, when parameters $P_{d y n}<0.3 \mathrm{nPa}, M_{A}<3$ (dashed line), and $\beta_{p}<0.1$. These are different from typical solar wind conditions at $1 \mathrm{AU}$, i.e. $M_{A} \geq 10, \beta_{p} \geq 1$, and $P_{d y n}=2.2 \mathrm{nPa}$.

When the density starts to recover after $\sim 00: 00$ UT (12), the interplanetary field and flow parameters are highly variable, and their magnitudes and amplitudes of variation are much larger (Fig. 2). The density and dynamic pressure approach steady values of $\sim 20 \mathrm{~cm}^{-3}$ and $\sim 5.5 \mathrm{nPa}$, respectively. These last $10 \mathrm{~h}$ contrast sharply with the preceding interval and are characterized by $M_{A} \geq 10$ and $\beta_{p} \geq 1$, as is typical of the solar wind at $1 \mathrm{AU}$ under, however, compressed conditions.

To summarize: Two types of solar wind are influencing the magnetosheath structure during the period of study: An extended segment where one would expect MHD effects to predominate, and another 2 segments (particularly the last $10 \mathrm{~h}$ ) where they should be much attenuated. Does the magnetosheath structure reflect this subdivision? Are there effects which may be attributed to the low $P_{d y n}$ and/or $M_{A}$ ? These are the questions we seek to answer.

We now correlate Wind and IMP 8 magnetic field data. IMP 8 was in the solar wind for long stretches of time, and it is located on the same side of the Sun-Earth line as Geotail (See Fig. 1). The highest cross-correlation coefficients for 10-12 May 1999, are 0.75( $\left.B_{x}\right), 0.77\left(B_{y}\right)$ and $0.76\left(B_{z}\right)$ reached at a time lag of $-3 \mathrm{~min}$, i.e. IMP 8 observed the same IMF features 3 min earlier than Wind. We may thus use Wind data. Below we shall assume a propagation time between Wind and Geotail of $0 \mathrm{~min}$. That this is a reasonable value may be seen from the light blue trace in the last panel of Fig. 2, which represents Geotail measurements of the clock angle, a quantity which correlates well across the bow shock (Song et al., 1992). With no lag assumed, it is seen that the agreement with Wind is very good up to $\sim 04: 00$ UT (12). A shift of $\sim 45 \mathrm{~min}$ appears on late 12 May since Wind and Geotail move in opposite directions. The large deviation at 06:00 UT (12) has other causes, as discussed in Sect. 3.2.1.

We next inquire why the measurements at Wind and Geotail may be considered as practically simultaneous for the earlier part of the interval under consideration. A minimum variance analysis of Wind magnetic field data (Sonnerup and Cahill, 1967) for the interval 10:00 UT (10)-04:00 UT (12) picks out a well defined normal. The ratio of intermediate-tominimum eigenvalues $=4.5$, and the field normal to the plane, $B_{n}=0.10 \pm 1.03 \mathrm{nT}$, consistent with zero. The normal to the plane is $\mathbf{n}=(0.601,0.644,0.474)$ (GSM coordinates). The sunward tilt of the plane explains why IMP 8 sees the solar wind before Wind, and Geotail at the same time as Wind, at least in the early part of the interval. We conclude that during the low-density event the IMF was to a good approximation a planar interplanetary structure containing the Parker spiral direction (Nakagawa et al., 1989). 

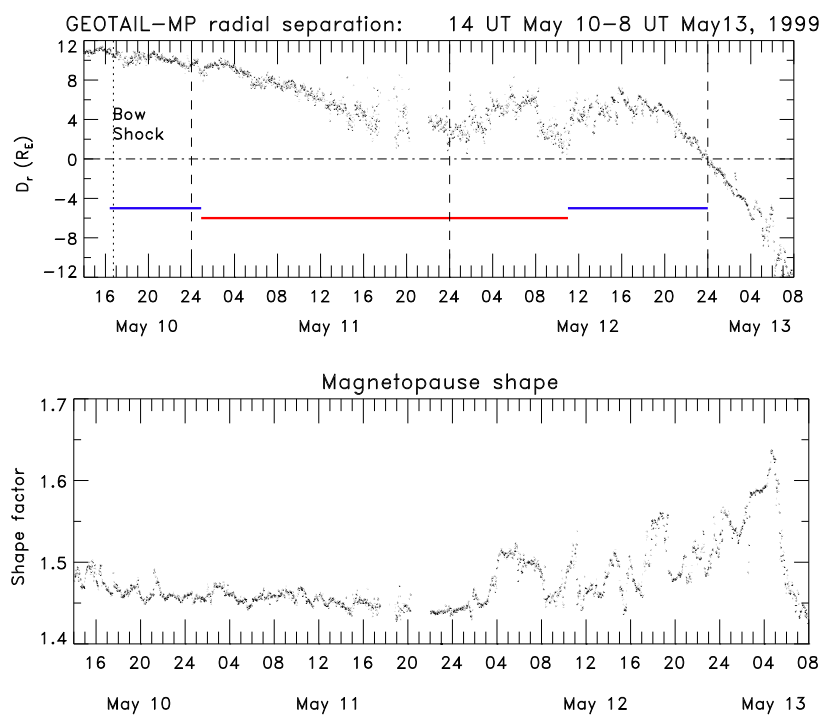

Fig. 4. For the period 14:00 UT (10) to 08:00 UT (13), the top panel shows the quantity $D_{r}$, defined as the radial distance of Geotail from the center of the Earth minus radial distance of the magnetopause. The time of the bow shock crossing is indicated by the vertical guideline on the left. The bottom panel plots the shape factor as a function of time, where the shape factor is defined as the terminator distance divided by the stand-off distance of the magnetopause. The model magnetosphere is that of Shue et al. (1998)

\section{Geotail observations}

\subsection{Position with respect to the magnetopause}

In order to interpret Geotail observations we first determine the spacecraft's location with respect to the magnetopause. We do this using the magnetopause model of Shue et al. (1998), where the magnetosphere shape is obtained as a function of both $P_{d y n}$ and IMF $B_{z}$.

The top panel of Fig. 4 shows the quantity $D_{r}$ defined as the distance of Geotail from the model magnetopause along the radial line. The Fig. extends from 14:00 UT (10) to 08:00 UT (13). When the solar wind density is decreasing, $D_{r}$ decreases monotonically on average, mainly as a result of the expansion of the magnetosphere. The spacecraft is several $R_{E}$ away from the model magnetopause, in the main body of the magnetosheath. As the pressure starts to recover, quantity $D_{r}$ increases. The close approach to the magnetopause during 08:00 UT (12)-11:00 UT (12) is a result of the drop in solar wind $P_{d y n}$ in this interval (Fig. 2). It is followed by a renewed magnetospheric compression. After 16:00 UT (12) when the pressure is high $(\sim 5.5 \mathrm{nPa})$ and fairly constant, $D_{r}$ decreases steadily, mainly as a result of the inward motion of the spacecraft (Fig. 1). According to the model, there should be a definitive magnetopause crossing at $\sim 00: 00$ UT (13). This value would be expected to be too early by $30-45 \mathrm{~min}$ because the zero-lag assumption breaks down on late 12 May and early 13 May as noted above.
The bottom panel of Fig. 4 gives an indication of the changing shape of the magnetopause. The "shape factor" plotted along the vertical axis is the ratio of the distance to the terminator to the subsolar stand-off distance in the equatorial plane, using the Shue et al. (1998) model. Throughout the density decrease, this quantity diminishes slowly, approaching a value of $\sim 1.44$ when $M_{A} \sim 1$. Thus according to the model the magnetosphere expands approximately selfsimilarly and reaches the quoted shape ratio when the bow shock is very weak/absent. The other extreme of vanishing IMF, i.e. gas dynamics, valid for $M_{A}->\infty$ (Spreiter et al., 1966), gives for the shape factor a value of 1.32 (Mead and Beard, 1964; see also Kivelson and Russell, 1995).

After $\sim 02: 00$ UT (12), as the density recovers in the second solar wind regime, and in particular the north-south component of the IMF undergoes large changes, the magnetospheric shape changes on short time scales and is in general blunter than before. Its most flared shape, at 05:00 UT (13) coincides with a dynamic pressure of $\sim 10 \mathrm{nPa}$, and a large, negative $B_{z}$ excursion (not shown).

\subsection{Geotail magnetosheath observations}

\subsubsection{Magnetic field and plasma}

Geotail magnetic field measurements from the MGF instrument at 3 s resolution (Kokubun et al., 1994) and plasma data from the LEP instrument at $64 \mathrm{~s}$ time resolution (Mukai et al., 1994) are shown in Fig. 5 for the period 14:00 UT (10) - 08:00 UT (13). From top to bottom the panels display the GSM components of the magnetic field, the total field, the proton density and temperature, the GSM components of the velocity vector, the total bulk flow speed, and the angle between the field and the velocity vectors. The green traces in the $B$ and $n_{p}$ panels reproduce, with zero time lag, the corresponding interplanetary measurements during the solar wind density decrease for comparison. The reader is also referred to Terasawa et al. (2000) for an overview of Geotail observations, and to Kasaba et al., (2000) for an overview of the Geotail electron observations.

A quasi-perpendicular bow shock $\left(\theta_{\mathbf{B}, \mathbf{n}}=84.5^{\circ}\right.$, the angle between the upstream IMF $\mathbf{B}$ and the normal $\mathbf{n}$ to the bow shock determined from the coplanarity theorem, see Abraham-Shrauner, 1972) is crossed at 16:40 UT (10). Thereafter, during the phase of decreasing density, the duskside magnetosheath density, temperature and magnetic field strength also decrease monotonically. The field and plasma variations become smooth as the density decreases. After 02:00 UT (12), when the solar wind density is recovering, magnetosheath field and plasma parameters display great variability. Thus these two magnetosheath states reflect the changes in the two solar wind regimes identified earlier. One may note the following features: (i) The approach of the ratios $n_{s h} / n_{s w}$ and $B_{s h} / B_{s w}$ to unity as the bow shock weakens, as noted by Terasawa et al. (2000); (ii) the approximately constant, small angle between field and flow in the first phase $\left(\leq 30^{\circ}\right)$; (iii) the increase in speed from 200 to $350 \mathrm{~km} \mathrm{~s}^{-1}$ 


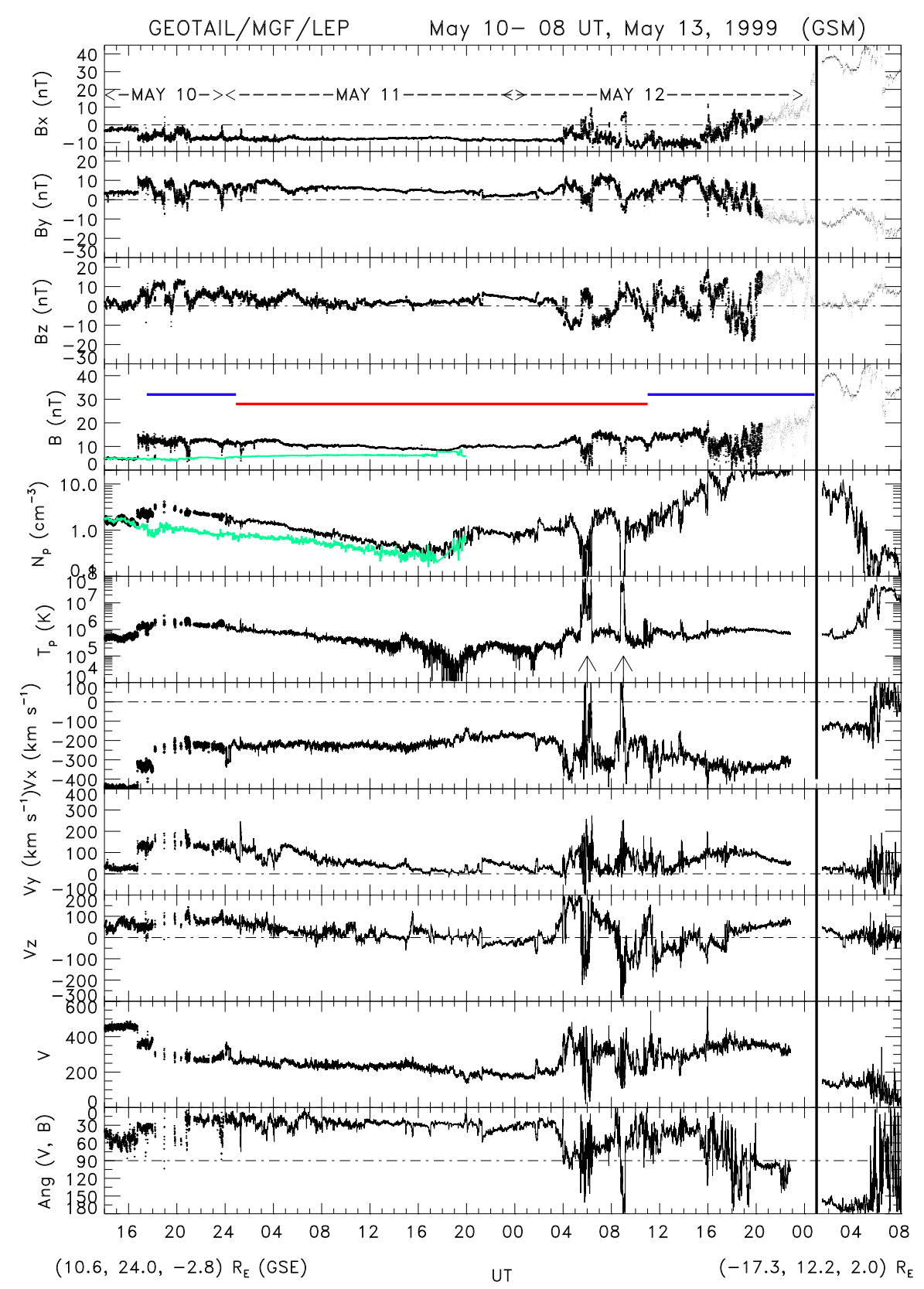

Fig. 5. Geotail field and plasma measurements for 14:00 UT (10)-08:00 UT (13) from the MGF and LEP instruments, respectively. From top to bottom the panels display the GSM components of the magnetic field, the total field, the proton density, temperature, the GSM components of the velocity vector, the total bulk flow speed, and the angle between the field and the velocity vectors. Overlaid as green traces are $B$ and $n_{p}$ panels from Wind.

and the deflection of the velocity at the start of the second phase. Similar changes occur in the solar wind and Geotail is thus observing a convected feature; (iv) impulsive changes in most parameters at what we shall show to be brief entries into the low latitude boundary layer (LLBL)/magnetosphere at around 06:00 UT (12) and 09:00 UT (12) (arrowed). Here the field is weak and the plasma is flowing sunward. (v) the bursts of compressive field oscillations, particularly evident during Geotail's final approach towards the magnetopause in the last $10 \mathrm{~h}$ (panels 1-4); (vi) the tailward stretched field encountered on entry into the magnetosphere (panel 1) as a precursor to a substorm onset which occurred at $\sim 04: 00$ UT (13) (Farrugia et al., 2000a); (vii) the definitive entry of the spacecraft into the LLBL at $\sim 01: 00$ UT (13), i.e. about $1 \mathrm{~h}$ after the entry predicted by the model assuming zero lag, and into the magnetosphere at $\sim 05: 30$ UT (13).

Other quantities of interest are shown in Fig. 6: the first 4 panels display the total field for reference, the proton plasma beta, the Alfven Mach number, and the 1-min averages of the proton temperature anisotropy, $A_{p}\left(A_{p}=T_{p, \perp} / T_{p, \|}-1\right)$. 

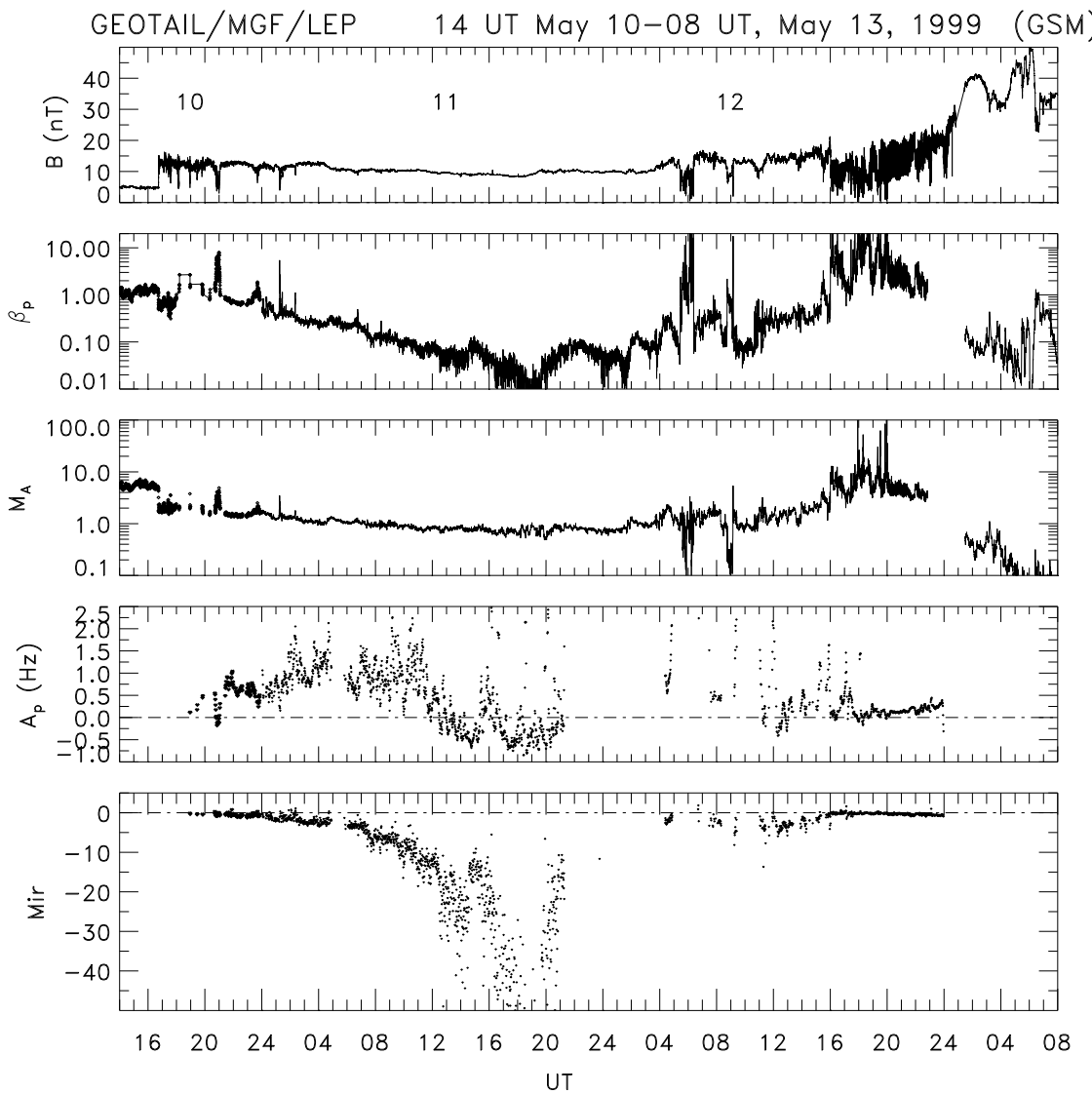

Fig. 6. For the same interval as Fig. 5, the panels show measurements by Geotail of the total field for reference, the proton beta, the Alfven Mach number, and the 1-min averages of the proton temperature anisotropy $\left(A_{p}=T_{p, \perp} / T_{p, \|}-1\right)$. The last panel plots the quantity $M i r$, defined in the text.

$T_{\perp}$ and $T_{\|}$are calculated from the ion moments by referring to the magnetic field direction. There is a gap in $A_{p}$ values from $\sim 20: 00$ UT (11) to $\sim 04: 00$ UT (12) because the magnetic field was directed in such a way that the parallel and perpendicular temperatures could not be reliably determined. The last panel plots the quantity Mir, defined by Mir $\equiv A_{p}-1 / \beta_{p, \perp}$. Condition Mir $\geq 0$ is necessary one for a mirror unstable configuration. The following points may be made:

1. The main body of the magnetosheath on 00:00 UT (11)16:00 UT (12), when Geotail is several $R_{E}$ from the magnetopause (red line in Fig. 4), is mirror stable; this is the opposite of the normal case.

2. Two large depressions in the field accompanied by a high $\beta_{p}$ and low $M_{A}$ mark the two brief entries into the LLBL/magnetosphere mentioned above.

3. The plasma beta is $<1$ in the MHD-dominated region. Thus the main body of the magnetosheath is PDL-like by definition (Sect. 1) (Farrugia et al., 1995).

4. Initially, the temperature anisotropy is positive with values $\leq 2.5$. As the pressure decreases further and the bow shock weakens, from $\sim$ 11:00 UT (11) onwards, $A_{p}$ decreases from values of 0.5 and becomes negative for some hours on either side of 16:00 UT (11), coinciding with the weakest bow shock.

This negative sign of $A_{p}$ is the same as in the solar wind at this time (see panel 5 in Fig. 2). Values of quantity Mir in the last portion of the pass, as the spacecraft approaches the magnetopause (where $\beta_{p}$ is generally $\gg 1$ and compressive magnetic fluctuations are observed) are approximately zero. Thus in the early and later part of the pass, the mirror mode is unstable, remaining close to the marginal limit.

We now direct attention to the two entries into the LLBL/magnetosphere at 06:00 UT (12) and 09:00 UT (12). Fig. 7 shows an expanded plot of the 5-h interval 05:0010:00 UT (12). The GSM location of the spacecraft is $(-8.8,27.5,5.7) R_{E}$ and $(-9.9,27.2,3.2) R_{E}$ at $05: 00$ and 10:00 UT (12), respectively. The panels are the same as those of Fig. 5, except that the last panel now shows the pressures: the field (black trace) and the proton temperature plasma pressure (red). The behavior of the particles (ions and electrons) is also shown in the spectrograms of Fig. 8. From top to bottom, this figure shows the omni-directional ion and electron fluxes, the electron fluxes travelling dawn- 


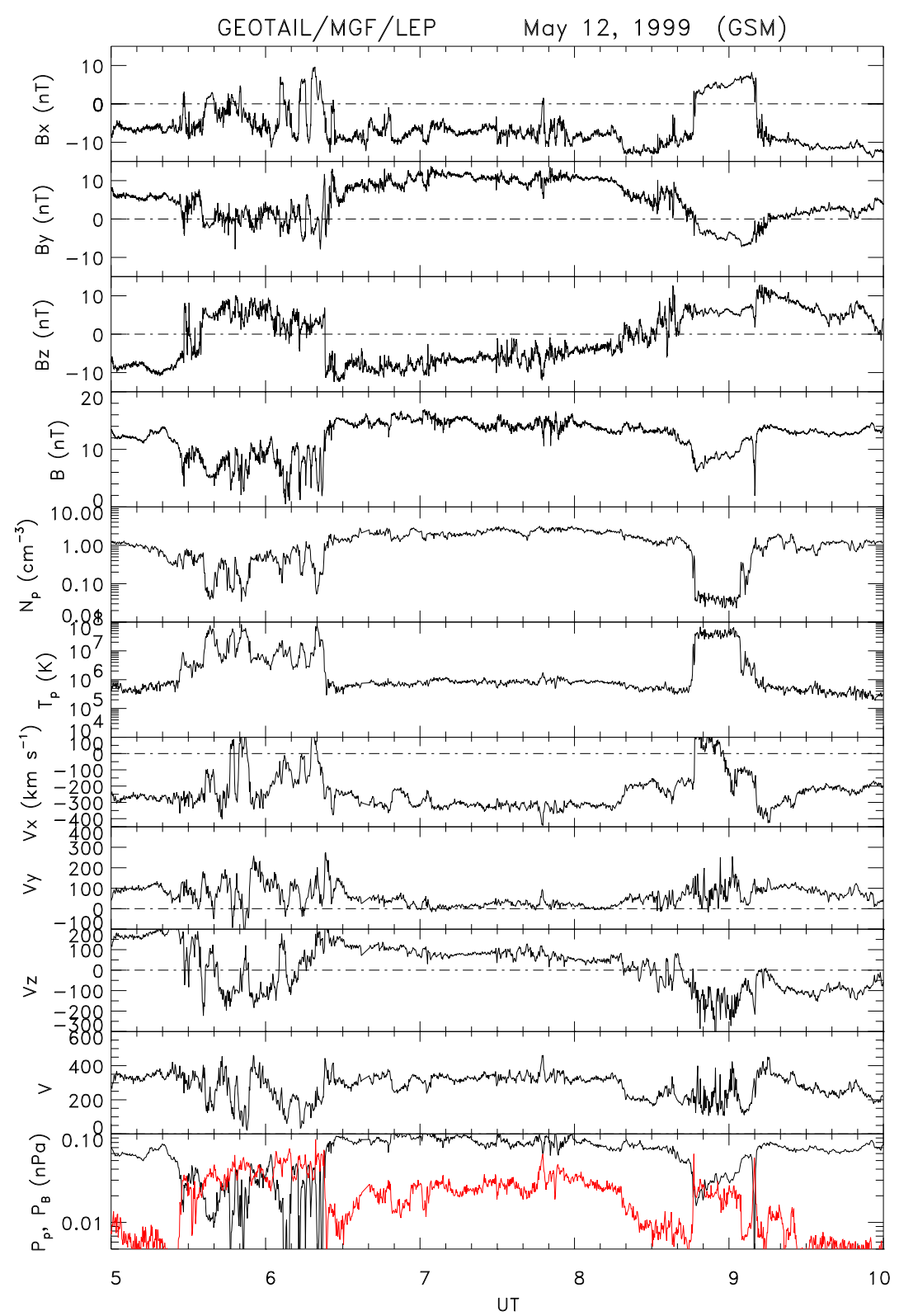

Fig. 7. Geotail measurements during 05:00 UT (12)-10:00 UT (12), in the same format as Fig. 5 except that the last panel gives the field (black trace) and proton plasma pressures.

ward, sunward, duskward and tailward, respectively, and ion fluxes of solar wind origin. Judging from the behavior of the proton density, temperature, and magnetic field strength and the spectral characteristics, the first entries are probably mostly into the LLBL because some low energy electron fluxes coexist with higher energy fluxes. At the inner edge of the LLBL, the flow is sunward (Figs. 7, 8), as predicted by Sonnerup (1980), see also Sonnerup and Siebert (2003). The second crossing is into the magnetosphere proper (absence of low energy electrons). Interesting in the first set of crossings is the way the magnetopause is kept in pressure balance. At these radial distances $\left(\sim 30 R_{E}\right)$, the magnetic field strength and magnetic pressure are very low compared to those in the magnetosheath (fourth and last panels of the Fig. 7), but the temperature is about 2 orders of magnitude higher, corresponding to the plasma sheet. Thus unlike the normal situation, at this inflated magnetosphere it is mainly the internal gas pressure which keeps the external pressure in check.

Compressional waves are only present for two short time segments of the pass when the proton plasma beta is large (Figs. 6, 7). Their average period is $16 \mathrm{~s}$, corresponding to a frequency, $f$, of $0.06 \mathrm{~Hz}$. According to theory, these waves are produced at zero frequency (Treumann and Baumjohann, 1997). If they are generated locally, the observed frequency $f$ would be $f \leq k_{\perp} V$, where $V \approx 400 \mathrm{~km} \mathrm{~s}^{-1}$ (Fig. 5), and we assumed the angle between flow and field to be $90^{\circ}$. For the wavelength perpendicular to the magnetic field $\left(\lambda_{\perp}\right)$, a rough upper limit is then $\lambda_{\perp} \leq 6.5 R_{E}$. 


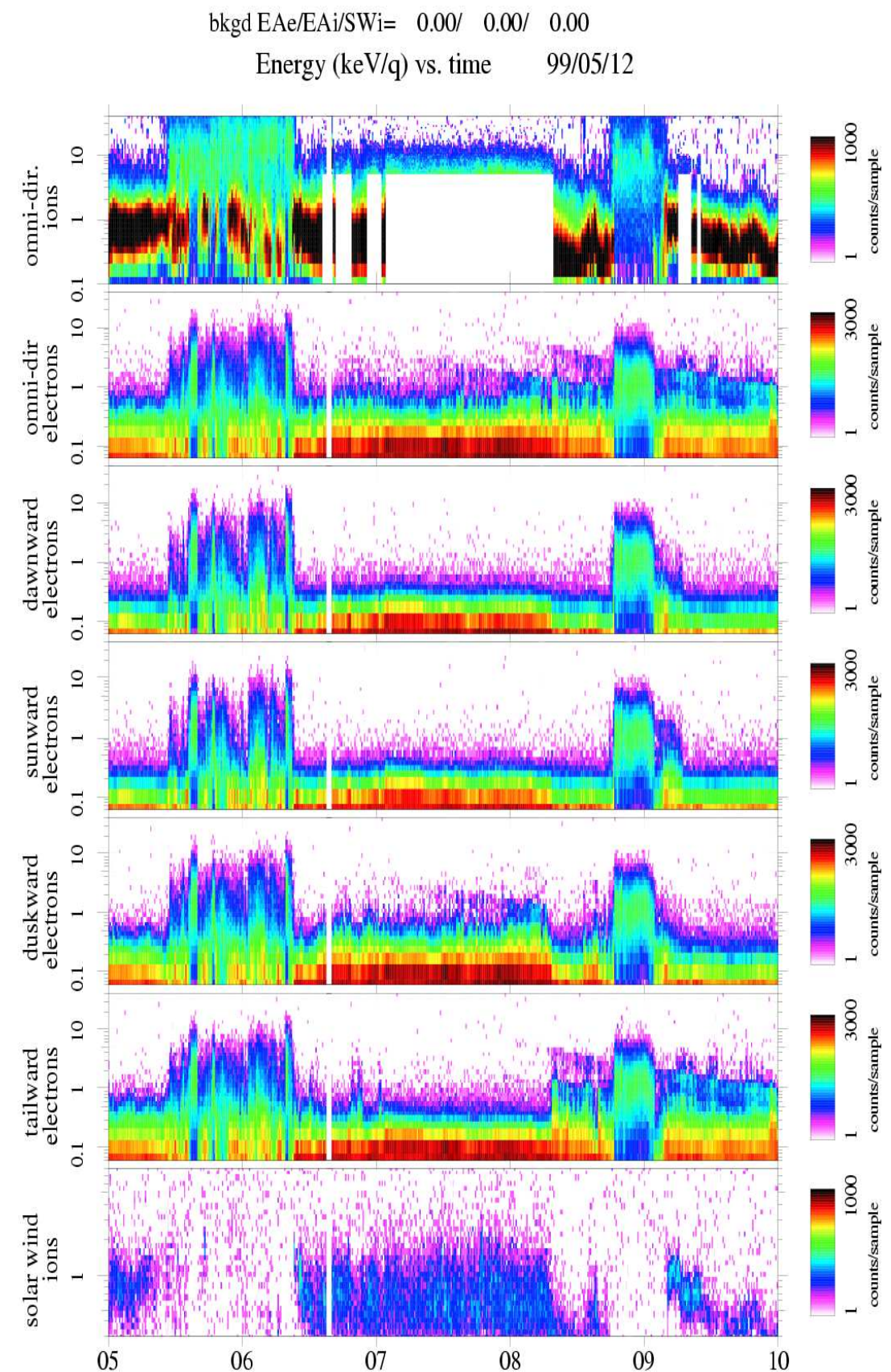

Fig. 8. From top to bottom, this figure shows Geotail measurements of the omni-directional ion and electron fluxes, the electron fluxes travelling dawnward, sunward, duskward and tailward, respectively, and ion fluxes of solar wind origin.

In the rest of the period under study, the total field is fairly steady, so that any waves have to be transverse to its direction. We study these waves next.

\subsubsection{Electromagnetic ion waves in the magnetosheath}

Figures 9, 10 and 11 show frequency-time spectrograms of the magnetic fluctuations for 10, 11 May and 12-02:00 UT (13), respectively. From top to bottom the panels display the spectral amplitude of right-hand $\left(B_{r}\right)$, left-hand $\left(B_{l}\right)$ and compressional $\left(B_{z}\right)$ components, color-coded according to the scale on the right. One Fourier transform is performed over 1024 data points. Each Fourier transform is shifted by 1024 points with respect to the previous one. The white trace in the middle panel gives the proton gyrofrequency in $\mathrm{Hz}$ presented as 5-point smoothed averages not to obstruct the wave data during intervals of strong fluctuations in $B$.

Quantites $B_{r}, B_{l}$, and $B_{z}$ are obtained as follows. The original magnetic field data from the on-board magnetometer are first despun. The $Z$ axis in the satellite coordinate system is along the spin axis, while the $X$ and $Y$ axes are in the 


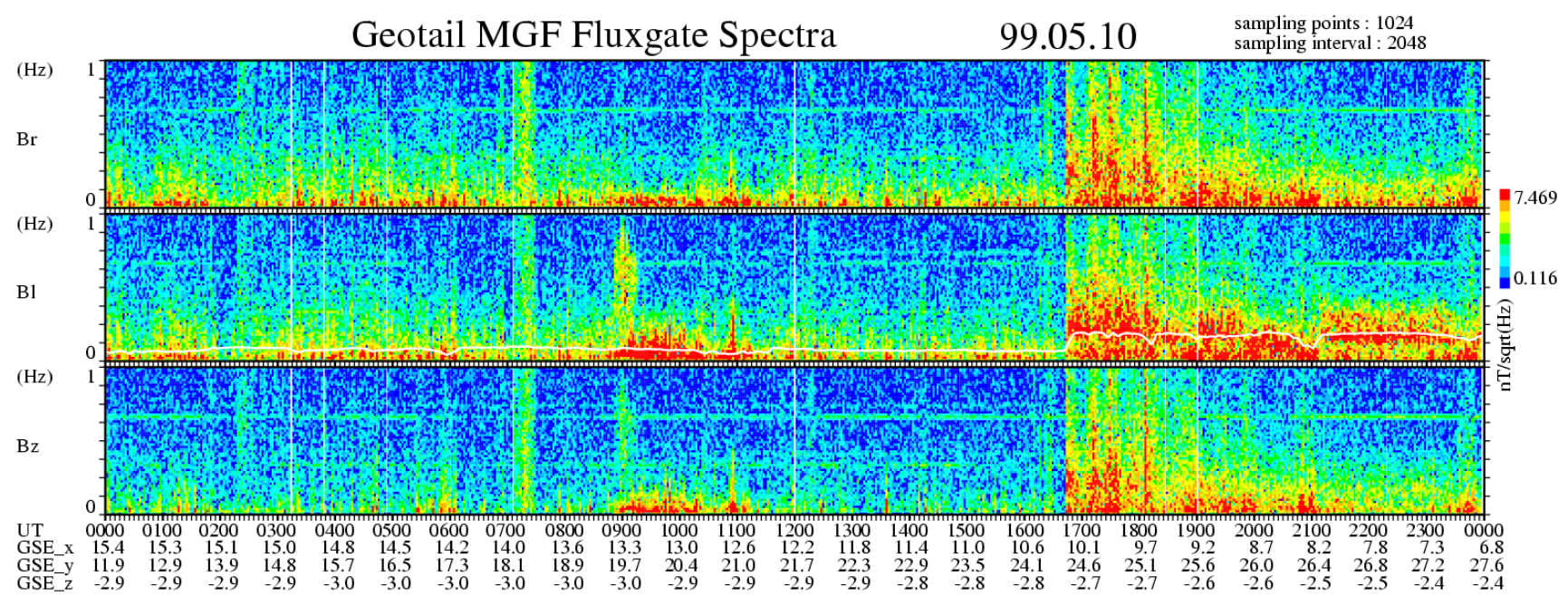

Fig. 9. Frequency-time spectrograms of the magnetic fluctuations obtained from Geotail measurements for 10 May. The panels show from top to bottom the power spectral amplitudes of right-hand $\left(B_{r}\right)$, left-hand $\left(B_{l}\right)$ and compressional $\left(B_{z}\right)$ waves, color-coded according to the scale on the right. The white trace in the middle panel gives the proton gyrofrequency in $\mathrm{Hz}$.

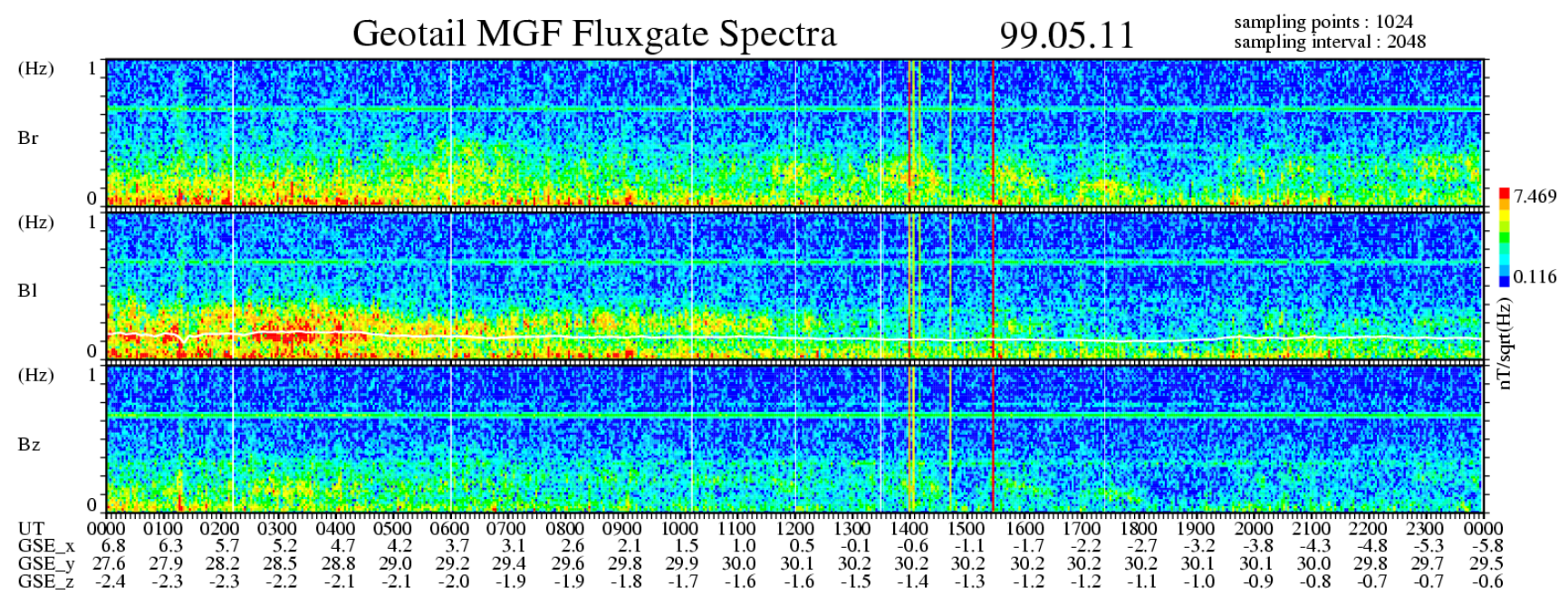

Fig. 10. Same as Fig. 9 but for 11 May 1999.

spin plane. This satellite coordinate system is close to the geocentric solar ecliptic (GSE) coordinate system, with the spin axis subtending an angle of $87^{\circ}$ to the ecliptic plane. The data are then transformed to a field-aligned coordinate system. In this system the $Z$ axis is parallel to the ambient magnetic field, the $Y$ axis is defined as the cross product of the unit vector in the direction of the spin axis and that in the direction of the ambient magnetic field, and the $X$ axis is defined by the cross product of unit vectors along the $Y$ and $Z$ axes. The right-hand and left-hand components of the magnetic field are defined as follows

$$
\begin{aligned}
& B_{X} \cos \left(\omega t+\phi_{B X}\right)+i B_{Y} \cos \left(\omega t+\phi_{B Y}\right) \\
& \quad=B_{r} \exp \left(i \omega t+\phi_{B r}\right)+B_{l} \exp \left(-i \omega t+\phi_{B l}\right)
\end{aligned}
$$

where $B_{X}$ and $B_{Y}$ are amplitudes of the $X$ and $Y$ components in the field-aligned system; $B_{r}$ and $B_{l}$ are the am- plitudes of the right-hand and left-hand components, respectively; and $\phi_{B X}, \phi_{B Y}, \phi_{B r}$, and $\phi_{B l}$ are phases for each component, respectively. The compressional component corresponds to the $Z$ component in the field-aligned system.

From 16:40 UT (10), when the bow shock was crossed, to 21:00 UT (10), wave power resides in both the compressional and transverse (to the background field) directions. Up to 19:00 UT (10) power is intermittently present up to frequencies of $\sim 1 \mathrm{~Hz}$ (for comparison, the proton gyrofrequency here is $\sim 0.18 \mathrm{~Hz}$ ), an upward shift in the frequency presumably due to the Doppler shift induced by the flow speed. After 21:00 UT, the compressional power fades slowly away (see also Fig. 6, bottom panel) and the frequency spectrum becomes dominated by left-handed transverse activity. This transverse activity continues until 12:00 UT (11). After $\sim 12: 00$ UT (11), as the bow shock becomes much weaker 


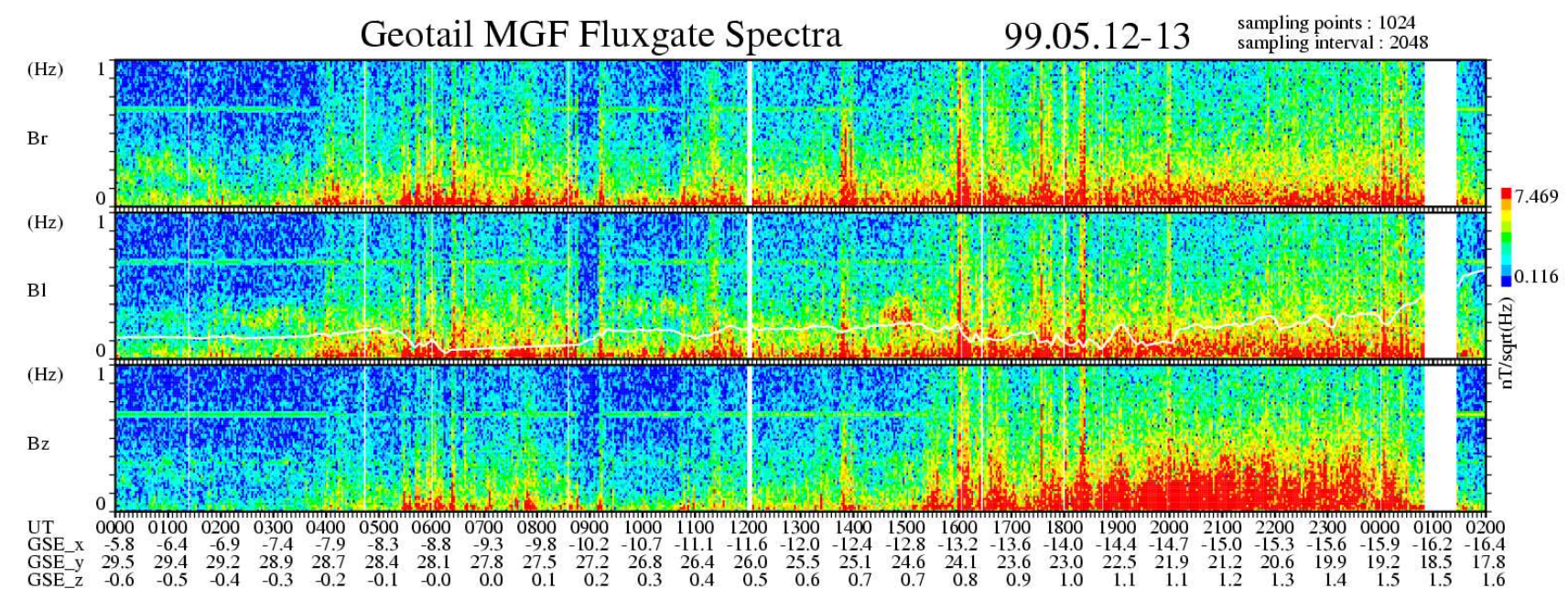

Fig. 11. Same as Fig. 9 but for 12 May-02:00 UT, 13 May 1999.

(Fig. 2) the left-hand power drops out, leaving only sporadic bursts of weak right-hand activity. This transition correlates well with significant values of $\left|A_{p}\right|$, with $A_{p}<0$ (Fig. 6).

On 12 May (Fig. 11c), as the density is recovering in the second solar wind regime, transverse and compressional wave activity resume between 04:00-09:00 UT. The bow shock is gaining in strength, increasing the perpendicular temperature and thereby the positive temperature anisotropy (Sckopke et al., 1990). During this period there are close encounters with magnetopause/LLBL. Indeed, during the entries identified above at 06:00 UT and 09:00 UT, the wave activity subsides, and in the second crossing drops out completely. Intense wave activity in all components resumes at $\sim 16: 00$ UT (12) with a predominance of the compressional power. This is the time when normal magnetosheath conditions prevail, as may be seen in the time series (Fig. 5).

Summarizing, during the density dropout (MHDdominated magnetosheath), the magnetosheath is bereft of waves, except for weak, sporadic, right-hand activity. In particular, compressional power in the main body of the magnetosheath is completely absent, the very reverse of what is typically seen at the dayside (Anderson et al., 1991, 1993) and on the flanks (Lucek et al, 1999, Farrugia et al., 2000b). Normal magnetosheath wave activity is present in the last 9$10 \mathrm{~h}$ of the pass. We now discuss the wave observations from the viewpoint of the linear kinetic theory of electromagnetic ion waves.

\section{Magnetosheath waves: theory}

The theory of electromagnetic ion waves is based on modes varying as $\exp (-i \omega t+i \mathbf{k} \cdot \mathbf{x})$, where a real wavevector $\mathbf{k}$ is given, and $\omega=\omega_{r}+i \gamma$ is in general complex valued. The real part $\omega_{r}=\Re(\omega)$ is the angular frequency in $\mathrm{rad} \mathrm{s}^{-1}$ of

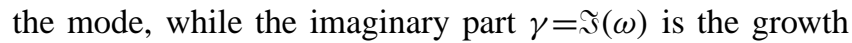
(damping) rate of the wave according as $\gamma>0(<0)$. Thus $\tau_{e}=1 / \gamma$ gives the corresponding e-folding time in s. Quan- tity $\omega$ is computed from a dispersion relation of the form $D(\omega, \mathbf{k}, Q)=0$ that contains a set of plasma parameters $Q$. This equation, derived from kinetic theory (see, e.g. Gary, 1993), is usually set up in the plasma frame. The waves can be excited by instabilities, depending on the values of the parameters $Q$.

In the frequency range $\omega_{r} \leq \Omega_{p}$ (the proton gyrofrquency), three wave modes were observed by Geotail in the magnetosheath, all of which are driven by $A_{p}$. Their intensity and other physical characteristics vary in concert with changes in $A_{p}$ and $\beta_{p}$, and are also Doppler-shifted by the flow $\mathbf{V}$, which near the dusk terminator is appreciable, to a frequency $\bar{\omega}_{r}=\omega_{r}+\mathbf{k} \cdot \mathbf{V}$. The waves were: 1) the mirror modes (MMs) 2) left-hand polarized ion cyclotron waves (L-EICWs) and 3) right-hand polarized ion cyclotron waves (R-EICWs). As noted in the Introduction, the MMs are usually the dominant wave mode in the main body of the (normal) magnetosheath, but disappear in the PDL because of the low $\beta_{p}$ prevailing in that region, see, e.g. (Schwartz et al., 1996). Because the R-EICWs are driven by a negative $A_{p}$, which is not usually the case in the magnetosheath, they are not ordinarily excited in the magnetosheath, and are observed there and in the PDL only as daughter waves, generated by nonlinear wave interactions in both regions.

Below we shall address the following points of the observations:

1. In comparison with typical EICW intensities exemplified by those on 10 May, EICWs in the low $P_{d y n}$-low $M_{A}$ magnetosheath are present but at much reduced intensities;

2. Sometimes R-EICWs appear alone driven directly by negative $A_{p}$; and

3. MMs are excited only in the high $M_{A}$ magnetosheath (at the beginning and end of the interval of interest), appearing together with EICW activity. 
We note that in the following calculations we omit the contribution of the $\alpha$-particles. We thereby underestimate the theoretical excitation rates of both L- and R-EICWs, which are both enhanced by the presence of $\alpha$ 's (see Gratton and Farrugia, 1996; Farrugia et al., 1998; Gnavi et al., 2000.) The same is true for MMs waves. The theory, therefore, gives only conservative estimates of the growth rates.

\section{$4.1 \quad$ L-EICWs}

The instability of L-EIC waves is due to positive $A_{p}$. These modes grow when the protons resonate with the waves, i.e. when condition

$\omega_{r}-k v_{\|}=\Omega_{p}$

is satisfied. Here, $v_{\|}$is the particle velocity parallel to the magnetic field. Waves propagating along $\mathbf{B}$, i.e. with $k_{\perp}=0$ and $k=k_{\|}$, are amplified faster, and we shall assume that the observed waves are mainly of this kind. We consider only protons. We shall also assume a bi-Maxwellian distribution,

$f_{B M a x}^{0}=\left(\frac{1}{\pi}\right)^{3 / 2} \frac{1}{v_{t h \|} v_{t h \perp}^{2}} \exp \left(-\frac{\left(v_{\|}\right)^{2}}{v_{t h \|}^{2}}\right) \exp \left(-\frac{\left(v_{\perp}\right)^{2}}{\left.v_{t h \perp}^{2}\right)}\right)$

where $\left.v_{t h, \|, \perp} \equiv \sqrt{(} 2 K_{B} T_{\|, \perp} / m_{p}\right)$.

It is convenient to normalize as follows:

$x=\omega / \Omega_{p}, \quad x_{r}=\omega_{r} / \Omega_{p}, \quad y=k V_{A} / \Omega_{p}, \quad g=\gamma / \Omega_{p}$.

The resonant protons move against the wave (i.e. with $v_{\|}<0$ when the phase velocity $v_{p h}>0$ ), so that resonance occurs for waves with $x_{r}<1$. At resonance (Eq. 2), wave emission or wave absorption may take place. However, when $x_{r}$ is less than a critical value $x_{c} \equiv A_{p} /\left(A_{p}+1\right)$, emission is the dominant process and the wave grows, while for $x_{r}>x_{c}$, absorption prevails. From equations $2-4$ it follows that the fraction of resonant protons is proportional to $\left.\exp \left(-\left(1 / \beta_{p, \|}\right)\left(\left(x_{r}-1\right) / y\right)\right)^{2}\right)$ (see, e.g. Gnavi et al., (2000), Farrugia et al. (2004), and references therein) and is thus regulated by $\beta_{p, \|} \equiv\left(v_{t h, \|} / V_{A}\right)^{2}$, decreasing as $\beta_{p, \|}$ decreases.

During May 11, 1999, L-EIWC activity was greatly curtailed by two factors: 1 ) the large decrease of $\beta_{p}$, which reduced the emission rate per particle, and 2) the strong decrease of the density which, in turn, reduced the number of emitters. Both effects are taken into account in the normalized growth rate, $g$, computed by solving for each real $y$ the dispersion equation of L-EICWs for $x$ (see, for instance, Gratton and Farrugia, 1996)

$y^{2}=A_{p}-x+\frac{\left(A_{p}+1\right) x-A_{p}}{y \sqrt{\beta_{\|, p}}} Z\left(\frac{x-1}{y \sqrt{\beta_{\|, p}}}\right)$.

$Z$ is the plasma dispersion function. In view of the fact that the electrons have only a minor influence on L-EICWs we have neglected the inertia of electrons and assumed that $A_{e}=0$ in (eq.5). (Wind/SWE values of $A_{e}$ lie in the interval $-0.5 \leq A_{e} \leq 0.5$ with large scatter).
Using experimental values of $A_{p}$ and $\beta_{\|, p}=\left(3 T_{\|} /\left(2 T_{\perp}+T_{\|}\right)\right) \beta_{p}$, we solve Eq. 5 with parameters changing with time. The normalized growth rates $g$ are shown as a function of the Doppler-shifted $x_{r}$ in the 3-D plots of Figs. 12 and 13. Time is the third axis and increases towards the left. Data for the calculations are available only where there is a horizontal line segment starting at the UT axis. When the horizontal line segment extends along the whole $x_{r}$ range the corresponding $g<10^{-4}$. From $\sim 21: 00$ UT (11) to $\sim 04: 00$ UT (12) there is a gap in the $A_{p}$ data and growth rates are not computed (blank in Fig. 13). The shift in frequency is computed and plotted only for forward-propagating waves $v_{p h}>0$ using the angle between $\mathbf{V}$ and $\mathbf{B}$ shown in the last panel of Fig. 5. We shall comment later about the Doppler shift of backward-propagating waves, which correspond to roots of the dispersion equation for negative $y$ values that are equally amplified. We can see that the frequency range of amplified waves goes intermittently beyond the proton gyrofrequency (at $x_{r}=1$ here, and $f_{p}$ in the spectrograms), as also observed in the spectrograms of Figs. 9 and 10, though experimental values are somewhat higher. Evidently there could be no agreement between theoretical and observed frequencies without taking into account the important Doppler shift at the spacecraft position.

The normalized frequency for which the theory predicts a maximum growth rate for L-EICWs is shown as a function of time in the upper panel of Fig. 14. The thin line joining plus symbols, computed with experimental data, is the frequency in the plasma frame, while the thick line joining diamond symbols corresponds to Doppler-shifted values for $v_{p h}>0$. The latter are about a factor of 2-3 higher than the former. The lower panel in Fig. 14 gives the corresponding normalized maximum growth rates as a function of time. The wide gap in computed properties, from $\sim$ 13:00 UT (11) to $\sim 04: 00$ UT (12) is due to the growth rates being less than $10^{-4}$ because of low $A_{p}$ and/or low $\beta_{p, \|}$, or absence of LEICWs amplification due to negative $A_{p}$, during the first part of the interval (up to $\sim 21: 00$ UT (11)), and for the rest of the period is due to the data gap noted above. A growth rate $g=10^{-4}$ is very weak, meaning that $\sim 1600$ proton gyroperiods have to elapse before the wave amplitude increases by a factor $e \simeq 2.72$.

Starting from Fig. 12 at 18:00 UT (10), theory shows EICWs amplification at $\sim 20: 00$ UT (10) followed by negligible $g$ values until a stronger excitation occurs at $\sim 21: 00$ UT (10), a time in which $A_{p}(>0)$ increases significantly (Fig. 6). The theoretical amplification is then modulated according to the variations of $A_{p}-\beta_{p}$ (Fig. 6), with varying values of $g$ from $\sim 21: 00$ UT (10) to $\sim 05: 00$ UT (11), when a short data gap appears. Additional modulations continue from $\sim 06: 00$ UT (11) to $\sim 10: 00$ UT (11), after which the excitation becomes negligible. We may note peaks of amplification, with $g$ between 0.01 to 0.1 at $\sim 21: 00$ UT (10), $\sim 02: 00$ UT (11), $\sim 04: 00-05: 00$ UT (11), $\sim 06: 00$ UT (11), $\sim 07: 00$ UT (11), 09:00 UT (11), and $\sim 10: 00-11: 00$ UT (11), separated by more or less wide and deep $g$ valleys with 
reduced wave excitation.

The absence of wave amplification from shortly after $\sim$ 11:00 UT (11) to after 20:00 UT (11) seen in Figs. 9 and also Fig. 14 is, according to the theory, a consequence of the long interval dominated by negative $A_{p}$ values, which prevented the excitation of L-EICWs (Fig. 6, panel 4). When positive $A_{p}$ were sporadically observed in this interval, the concomitant low beta was not sufficient to produce $g>10^{-4}$.

Figure 13, which is a continuation of Fig. 12, covers the period from 12:00 UT (11) to 20:00 UT (12). Here the amplification reappears at $\sim 04: 00$ UT (12), after the data gap. The growth rate rises to a peak $g \sim 0.1$ at $\sim 05: 00$ UT (12), it is negligible from $\sim 07: 00-08: 00$ UT (12), grows again to substantial values from $~ 09: 00-11: 00$ UT (12), falls down after $\sim$ 11:00 UT (12) and peaks strongly again at 12:00 UT (12). Therafter follows a valley of very low $g$ up to $\sim 15: 00$ UT (12), then some additional peaks at $\sim 15: 00-16: 00$ UT (12), and at $\sim 17: 00$ UT (12), after which the theoretical amplification becomes negligible.

After about 18:00 UT (12), during the high $M_{A}$ phase brought about by the recovery of the density, the compressional activity observed in the spectrograms (which restarted some hours earlier) becomes stronger and keeps $A_{p}$ at low values, as shown in Fig. 6 . The small $A_{p}$ values, in turn, reduce the growth rate of the L-EICWs to negligible values, as shown in Figs. 10 and 11, so that we do not extend Fig. 13 beyond 20:00 UT (12).

\subsection{R-EICWs}

The R-EICWs are observed sometimes together with LEICWs, and at other times alone (Figs. 9, 10 and 11). These two cases have to be treated separately.

According to linear theory, an instability of the R-EICWs can be excited by a negative temperature anisotropy, provided the $\beta_{p}$ is not too small, (see (Gary, 1993). At the same time, a negative $A_{p}$ inhibits the growth of the L-EICWs, as noted above. According to the linear theory R-EIC waves are not amplified at all when $A_{p}>0$. When R-EICWs are observed under $A_{p}>0$ conditions, they are by-products of LEIC waves (see, for example, last part of 10 May and early part of 11 May). Note also that when $A_{p}>0$ and $\beta_{p} \leq 1$ the R-EICWs have little or no damping, particularly at $x_{r}<1$, so they may last long after being generated. Conversely, when $\beta_{p}$ is large the damping of R-EICWs may increase considerably, except at very low frequencies, $x_{r} \ll 1$.

We consider now the case of negative $A_{p}$. Geotail observes weak bursts of R-EICWs with little accompanying L-EICWs (Fig. 10). These right-hand waves are now being generated directly from the temperature anisotropy. Interestingly, this period coincides with a weak bow shock so that the magnetosheath is exposed to the proton temperature anisotropy of the solar wind. This is negative (see Fig. 2, panel 5). We conclude that this weak R-EICW activity is directly a result of the weakening of the bow shock, thus eliminating a major source for preferentially enhancing $T_{\perp}$ at the expense of the $T_{\|}$(Sckopke et al., 1990).

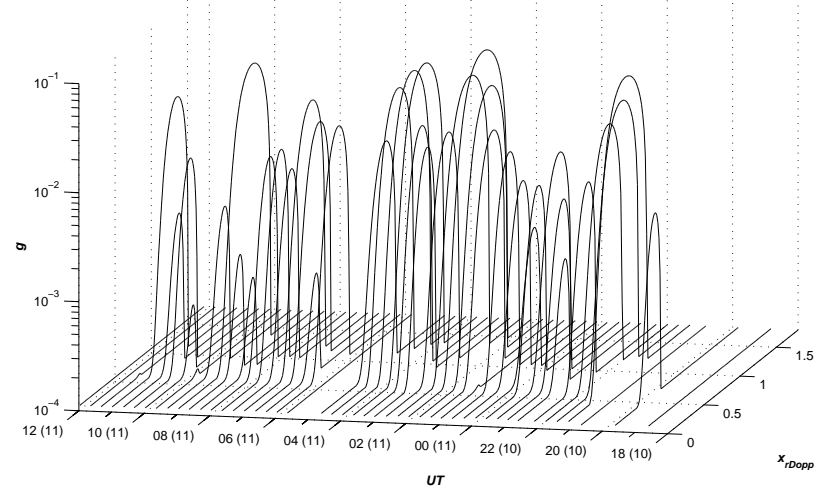

Fig. 12. Theoretical results on L-EICWs using measured values at Geotail. Plotted are the growth rates, $g$ in units of $\Omega_{p}$, against UT and Doppler - shifted frequecies $x_{r}$ normalized to $\Omega_{p}$. The time axis is in hours starting from 18:00 UT, 10 May 1999, and increases to the left. This interval starts soon after the bow shock crossing and ends at the lowest values of the solar wind density 12:00 UT (11). Left-hand-amplification with modulations in $g$ throughout the interval except for $2 \mathrm{~h}$ at its extremities.

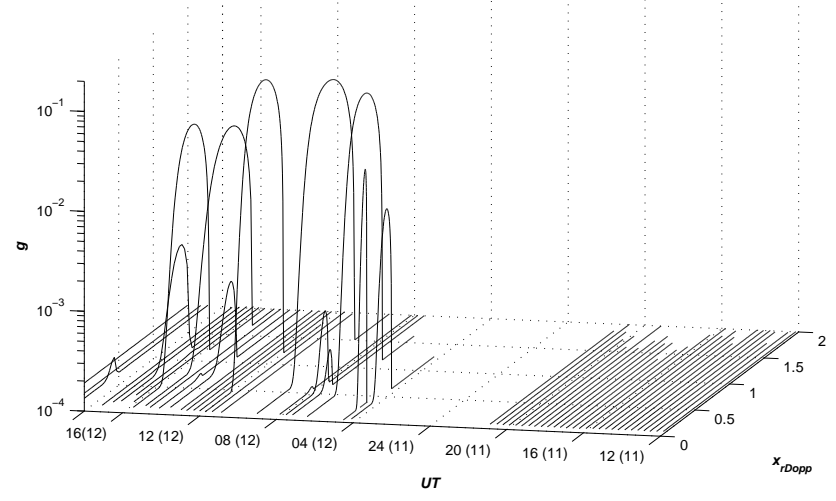

Fig. 13. Same as Fig. 9, but for the interval 12:00 UT (11) to 20:00 UT (12), i.e. during the phase when the density starts to recover. Note the absence of L-EICW amplification in the interval 12:00 UT (11) to 04:00 UT (12), in agreement with the data (Fig. 10).

The R-EICW activity observed in the interval after $\sim 12$ UT (11) and $\sim 21: 00$ UT (11) when $A_{p}<0$ corresponds to very low values $\beta_{p}(<0.1)$, but we must note that $\beta_{p, \|}$, which is the key parameter for the waves, was $>0.1$ due to the sporadically enhanced, negative anisotropy. As an example of the theoretical results, we quote two computations, one for $\sim 12: 00$ UT (11) and the other for 15:00-16:00 UT (11). (For the dispersion relation see, for example Farrugia et al., 1998). For the first we have $\beta_{p}=0.09$ and $A_{p}=-0.8$, and $\beta_{p, \|}=0.19$. For the second we have $\beta_{p}=0.09, A_{p}=-0.94, \beta_{p, \|}=0.24$. In both cases we find $g \approx 0.1$ in the frequency range $0.35-0.37$ $\Omega_{p}$. A growth rate $g=0.1$ is important (every $\sim 1.6$ proton gyroperiods, there is an e-folding rise of the wave amplitude). When the quoted frequency is Doppler-shifted by a factor 2- 

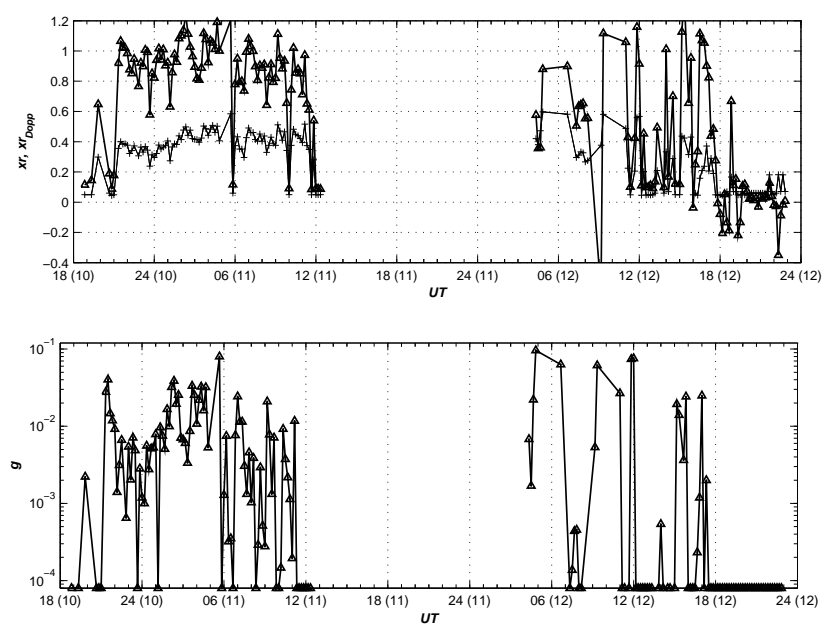

Fig. 14. Theoretical maximum growth rates of L-EICWs (bottom panel) and corresponding frequencies (upper panel) for the interval 18:00 UT (10)-24:00 UT (12), where data for the computations are available. Note that at dusk there is a large Doppler shift in the frequency (thick line) due to the fast magnetosheath flow at dusk. In the gap the temperature anisotropy $A_{p}<0$, and these waves are not excited.

3 as before, it yields for $v_{p h}>0$ a frequency $\sim \Omega_{p}$. Outside the cited frequency range the growth rate becomes very small because $\left|A_{p}\right|$ is small. These estimates are in agreement with the observed weak right-handed emission when $A_{p}<0$.

The weakness of the R-EICW power at this time may be explained as follows. The amplification of R-EICWs is due to the cyclotron resonance of ions with the waves:

$\omega_{r}-k v_{\|}=-\Omega_{p}$,

which is known as the anomalous resonance condition. Equation (eq-6) is satisfied only when $v_{\|}>v_{p h}$, and for these waves $v_{p h}>V_{A}$. Therefore, under this condition, and remembering that $\beta_{p, \|}=\left(v_{t h, \|} / V_{A}\right)^{2}$, it follows that there are only few particles in the proton distribution function that travel faster than the R-EIC waves when $\beta_{p, \|} \ll 1$ and resonate, since $v_{p h}>V_{A}>v_{t h}$. Therefore, as $\beta_{p, \|}$ decreases due to an increase of $V_{A}$ at nearly constant temperature, the R-EICWs excitation is reduced.

\subsection{Mirror mode waves}

The mirror modes have a wavevector quasi-perpendicular to the field $\left(k_{\perp} \gg k_{\|}, k \approx k_{\perp}\right)$. A characteristic signature of the MMs is the anticorrelation of magnetic field fluctuations with density perturbations. The condition for the MMs instability resulting from kinetic theory is

$1-\sum_{s}\left(\beta_{\perp, s} A_{s}\right) \leq 0$,

where the sum extends over all the particle species (see, e.g. Hasegawa, 1975; Treumann and Baumjohann, 1997).

Taking only protons into account, we represent the theoretical instability limit (Eq. 7) by a horizontal line in last panel of Fig. 6. The points represent experimental values. The quantity $\beta_{p}$ and the temperature ratios are 1-min running averages. The mirror instability is a non-resonant process in which all the particles participate, and it tends to reduce the anisotropy as the amplitude of the waves grows. Therefore, these modes are ordinarily only marginally unstable, and are observed close to the theory limit.

\subsection{Comparison of wave theory with the observed spectra}

A general preliminary comment: the linear theory of waves cannot reproduce fully the observed power spectrum, due to the absence of wave-wave interactions and other realistic elements omitted in the treatment. Nevertheless, the linear model can point out some basic trends of the wave activity, predict the presence or absence of the instability, and reveal the influence on the waves of the variation of important physical parameters. With these limitations in mind, we carry out a qualitative comparison of the theoretical properties computed with the observed wave phenomena.

Between $~ 21: 00$ UT (10) and 11:00 UT (11) a qualitative agreement between the observed spectral activity (Figs. 9, 10) and the computed frequencies and amplification rates can be noted. (see Figs. 12 and 14) At about 21:00 UT (10), when the mirror mode activity declines significantly, the parameter $A_{p}$ increases and, correspondingly, the growth rates of L-EICWs expected from linear theory reach substantial values (Fig. 12). Note also in Figs. 9-10 the observed relative reduction of L-EICWS activity at about and after 24:00 UT (10), a recovery from 02:30 to 04:30 UT (11), a decline from 05:00 UT (11) to 07:00 UT (11), followed by a weak revival at low frequencies from 07:00 UT (11) to 11:00 UT (11), and the subsequent fade out. These features are qualitatively reflected in Figures 12 and 14 as trends of growth rate variation, at approximately the same times.

The observed intensity at very low frequencies, with $\omega \ll \Omega_{p}$ in the range $\mathrm{f}<0.1 \mathrm{~Hz}$ (Figs, 9, 10 and 11) can be explained within the linear theory by the amplification of backward-propagating L-EICWs $\left(v_{p h}<0\right)$. For these waves, which propagate against the field, $k_{\|}$is replaced by $-k_{\|}$, and the observed resonant frequency is, therefore, $\bar{\omega}_{r}=\omega_{r}-|k| V$ (Eq. 2) so that the Doppler shift may reduce their frequency considerably. An additional, different, contribution to the LEIC wave population at low frequency may be non-linear interactions producing a cascade from the excited frquency range down to lower frequencies.

A temporary absence of theoretical L-EICW growth rate may be noted at $\sim 09: 00$ UT (12), Fig. 13, at the same time of a transient entry of Geotail into the magnetosphere, discussed before, when a power decline appears in the spectrogram of Fig. 11.

The presence of R-EICWs under positive temperature anisotropy conditions seen in the spectrograms can be explained by the linear theory, from the Doppler shift of already existing backward propagating L-EICWs, or those excited together with forward propagating L-EICWs when $A_{p}>0$. We have seen (Fig. 14) that the Doppler shift may 
increase the frequency of L-EICWs with $v_{p h}>0$ by factors varying from $2-3$. For $v_{p h}<0$, negative values of $\bar{\omega}_{r}$ are also obtained, which means that Doppler-shifted, backward propagating L-EICWs can be observed as R-EICWs. The recorded R-EICWs may also be generated, at least in part, by nonlinear wave interactions that can produce these modes from pre-existing L-EIC waves.

Theory predicts no wave amplification from $\sim 11: 00$ UT (11) to $\sim 21: 00$ UT (11) (Figs. 12-13), a long interval where available $A_{p}$ data shows a preponderance of negative values, which prevent the growth of L-EIC waves. The same time period is characterized by a sharp decline, and almost absence of L-EICW power, except for a trace at very small frequencies (Fig. 11). This is also the period of observed weak bursts of R-EICWs power, in agreement with theoretical notions about R-EICWs excitation when $A_{p}<0$. The R-EICW bursts occur at about 12:00 UT (11), 14:00 (11), 16:00 (11), 18:00 (11), 20:00 (11), and 21:00 UT (11). As noted, this activity cannot be a by-product of L-EICWs excitation.

The agreement between the theoretical instability limit of Fig. 6 and the MMs activity shown in the spectrograms of Figs. 9-11 is, in general, good during the whole interval studied, taking into account the fact that mirror modes are ordinarily only marginally unstable, as mentioned in the Introduction and Sect. 4.3. This comment applies also to some details of the spectrograms. For instance, one may note the MM bursts at $\sim 21: 00$ UT (10), and at $\sim 06: 00$ UT (12) that can be correlated with points lying on, or above, the theoretical instability limit at the same times.

To conclude, not all the observed spectral features can be explained by the linear theory. An example of the latter discrepancy is the L-EICW activity seen in Fig. 11 after $\sim 18: 00$ UT (12). Furthermore, the critical quantity $A_{p}$ is not easily measured, and has been 1 min averaged. Nevertheless we have been able to point out several qualitative, sometimes even quantitative, agreements between theory and experiment.

\section{Conclusion}

We have presented observations of magnetosheath field, plasma, and, most of all, wave properties during very unusual conditions. We now highlight what, in our view, is the significance of the reported results on our knowledge of the magnetosheath. The major extreme element was the very low solar wind dynamic pressure. This had two consequences. This first is that, because at the same time the magnetic field strength remained fairly constant, the solar wind Alfven Mach number was also low. The latter implies that magnetosheath properties were controlled strongly by the IMF, which means that the magnetosheath was essentially PDLlike. An expectation of both theory and observations concerning this regime is that there should be transverse wave activity, EICWs. In fact, these were observed. Thus we have verified a theoretical expectation, and extended observational work on electromagnetic ion waves in the magnetosheath to the low dynamic pressure regime.

The second consequence of low solar wind dynamic pressure is that the resulting Alfven (and magnetosonic) Mach number weakened the bow shock. The weak bow shock exposed the magnetosheath directly to the temperature anisotropy of the solar wind, which was negative. Plasma wave theory then predicts the presence of right-hand polarized EICWs, deriving their energy from the anisotropy. And these waves were observed. We have thus confirmed another prediction of wave theory in the context of space physics plasmas. To our knowledge, this is the first observation of transverse right-hand EICWs existing alone in the magnetosheath.

The temporal aspect of the observations was very crucial in our case. As Fig. 3 clearly demonstrates, the solar wind underwent a large-scale transformation, and Geotail stayed long enough in the magnetosheath to observe its effect. In this case, clearly, what class of waves were observed depended on a temporal feature and did not reflect a spatial structure of the magnetosheath. As a corollary, the waves observed were not related to the position of the spacecraft with respect to the magnetopause or bow shock: EICWs were seen in the main body of the magnetosheath, without mirror waves; and mirror mode waves were observed right next to the magnetopause.

Acknowledgements. Part of this work was done while FTG and GG were on research visits at the Space Science Center of the University of New Hampshire. We thank T. Nagai for kindly providing the magnetic field data from Geotail. Geotail data are courtesy of the DARTS website at http://www.darts.isas.ac.jp. This work is supported in part by NASA Grants NAG5-12189, and NAG5-13116, NASA Wind Grant NAG5-11803, and the Argentinian UBACyT grant X032 and CONICET PIP 2013/01.

Topical Editor T. Pulkkinen thanks C.-H. Lin for his help in evaluating this paper.

\section{References}

Abraham-Shrauner, B.: Determination of magnetohydrodynamic shock normals, J. Geophys. Res., 77, 736-739, 1972.

Anderson, B. J. and Fuselier, S. A.: Magnetic pulsations from 0.1 to $4 \mathrm{~Hz}$ and associated plasma properties in the Earth's subsolar magnetosheath and plasma depletion layer, J. Geophys. Res., 98, 1461-1480, 1993.

Anderson, B. J., Fuselier, S. A., and Murr, D.: Electromagnetic ion cyclotron waves observed in the plasma depletion layer, Geophys. Res. Lett., 18, 1955-1958, 1991.

Anderson, B. J. Fuselier, S. A., Gary, S. P., and Denton, R. E.: Magnetic spectral signatures in the Earth's magnetosheath and plasma depletion layer, J. Geophys. Res., 99, 5877-5892, 1994.

Erkaev, N. V. : Results of the investigation of MHD flow around the magnetosphere, Geomagn. Aeron., 28, 455-464, 1988.

Fairfield, D. H.: Waves in the vicinity of the magnetopause, in: Magnetospheric Particles and Fields, McCormac, B. M. (Ed.), D. Reidel Pub. Comp., Dordrecht, Holland, 67-76, 1976. 
Fairfield, D. H., Cairns, I. A., Desch, M. D., Szabo, A., Lazarus, A. J., and Aelig, M. R.: The location of low Mach number bow shocks at Earth, J. Geophys. Res., 106, 25 361-25 376, 2001.

Farrugia, C. J., Erkaev, N. V., Biernat, H. K., and Burlaga, L. F.: Anomalous magnetosheath properties during Earth's passage of an interplanetary magnetic cloud, J. Geophys. Res., 100, 19245$19258,1995$.

Farrugia, C. J., Gratton, F. T., Gnavi, G., and Ogilvie, K. W.: On the possible excitation of electromagnetic ion cyclotron waves in ejecta, J. Geophys. Res., 103, 6543-6550, 1998.

Farrugia, C. J., Singer, H. J., Evans, D. S., Berdichevsky, D., Scudder, J. D., Ogilvie, K. W., Fitzenreiter, R. J., and Russell, C. T.: Response of the equatorial and polar magnetosphere to the very tenuous solar wind on 11 May 1999, Geophys. Res. Lett. 27, 3773-3776, 2000a.

Farrugia, C. J., Gratton, F. T., Contin, J., Cocheci, C. C., Arnoldy, R. A., Ogilvie, K. W., Lepping, R. P., Zastenker, G. N., Nozdrachev, M. N., Fedorov, A., Sauvaud, J.-A., Steinberg, J. T., and Rostoker, G. : Coordinated Wind, Interball/tail, and Ground observations of Kelvin-Helmholtz Instability and Waves in the near-tail, equatorial magnetopause at dusk: 11 January 1997, J. Geophys. Res., 105, 7639-7668, 2000b.

Farrugia, C. J., Gnavi, G., Gratton, F. T., Matsui, H., Torbert, R. B., Lepping, R. P., Oieroset, M., and Lin, R. P.: Electromagnetic ion cyclotron waves in the subsolar region under normal dynamic pressure: Wind observations and theory, J. Geophys. Res., 109, doi: 10.1029/2003JA010104, 109, 2004.

Gary, S. P.: Theory of Space Plasmas Microinstabilities, Cambridge University Press, New York, 1993.

Gary, S. P. and Lee, M. A.: The ion cyclotron anisotropy instability and the inverse beta correlation between proton anisotropy and proton beta, J. Geophys. Res., 99, 11 297-11 302, 1994.

Gary, S. P., McKean, M. E., Winske, D., Anderson, B. J., Denton, R. E., and Fusilier, S. A. : Proton cyclotron anistropy instability and the anisotropy/beta inverse correlation, J. Geophys. Res., 99, 5903-5914, 1994.

Gnavi, G., Gratton, F. T., and Farrugia, C. J.: Theoretical properties of electromagnetic ion cyclotron waves in the terrestrial, dayside, low latitude plasma depletion layer under uncompressed magnetosheath conditions, J. Geophys. Res., 105, 20 973-20 988, 2000.

Gratton, F. T. and Farrugia, C. J., Electromagnetic ion cyclotron waves in the terrestrial plasma depletion layer: Effects of possible relative motion between $\mathrm{H}+$ and $\mathrm{He}^{2+}$ ions, J. Geophys. Res., 101, $21553-21560,1996$.

Hasegawa, A.: Plasma Instabilities and Non Linear Effects, Springer Verlag, New York, 1975.

Hill, P., Paschmann, G., Treumann, R. A., Baumjohann, W., and Sckopke, N. : Plasma and magnetic field behavior across the magnetosheath near local noon, J. Geophys. Res., 100, 95759584, 1995.

Kasaba, Y., Terasawa, T., Tsubouchi, K., Mukai, T., Saito, Y., Matsumoto, H., et al. : Magnetosheath electrons in anomalously low density solar wind observed by Geotail, Geophys. Res. Lett., 27, 3253-3256, 2000.

Kivelson, M. G. and C. T. Russell, (Eds) : Introduction to Space Physics, Cambridge University Press, New York, 1995.

Kokubun, S., Yamamoto, T., and Acuna, M. H., et al.: The Geotail Magnetic Field Experiment, J. Geomag. Geoelectr., 46, 7-21, 1994.

Lees, L.: Interaction between the solar wind plasma and the geomagnetic cavity, AAIA J, 2, 1576-1582, 1964.

Lepping, R. P., Acuna, M. H. and Burlaga, L. F., et al.: The
Wind Magnetic Field Investigation, Space Sci. Rev., 71, 207229, 1995.

Lucek, E. A., Dunlop, M. W., Balogh, A., Cargill, P., Baumjohann, W., Georgescu, E., Haerendel, G., and Fornacon, H.-K.: Identification of magnetosheath mirror modes in Equator-S magnetic field data, Ann. Geophys., 17, 1560-1573, 1999.

Matsui, H., Farrugia, C. J., and Torbert, R. B.: Wind-ACE solar wind correlations, 1999: An approach through spectral analysis, J. Geophys. Res., 1355, doi:10.1029/2002JA009251, 107, 2002.

Midgley, J. E. and Davis, L.: Calculation by a moment technique of the perturbation of the geomagnetic field by the solar wind, J. Geophys. Res., 68, 5111-5123, 1963.

Mead, G. D. and Beard, D. B. : Shape of the geomagnetic field solar wind boundary, J. Geophys. Res., 69, 1169-1179, 1964.

Mukai, T., Machida, S., and Saito, Y., et al.: The Low-Energy Particle (LEP) Experiment onboard the Geotail satellite, J. Geomag. Geoelectr., 46, 669-692, 1994.

Nakagawa, T., Nishida, A., and Saito, T.: Planar magnetic structures in the solar wind, J. Geophys. Res., 94, 11 761-11 775, 1989.

Ogilvie, K. W., Chornay, D. J., and Fitzenreiter, R. J., et al.: SWE, A comprehensive plasma instrument for the Wind spacecraft, Space Sci. Rev., 71, 55-77, 1995.

Phan, T.-D., Paschmann, G., Baumjohann, W., Sckopke, N., and Luehr, H.: The magnetosheath region adjacent to the dayside magnetopause: AMPTE/IRM observations : J. Geophys. Res., 99, 121-142, 1994.

Richardson, J. D. and Paulerena, K. I.: Plasma and magnetic field correlations in the solar wind, J. Geophys. Res., 103, 239-252, 2001.

Schwartz, S. J., Burgess, D., and Moses, J. J.: Low-frequency waves in the Earth's magnetosheath: present status, Ann. Geophys., 14, 1134-1150, 1996,

SRef-ID: 1432-0576/ag/1996-14-1134.

Shue, J.-H., Song, P., Russell, C. T., Steinberg, J. T., Chao, J. K., Zastenker, G., Vaisberg, O. L., Kokubun, S., Singer, H. J., Detman, T. R., Kawano, H.: Magnetopause location under extreme solar wind conditions, J. Geophys. Res., 103(A8), 17 691-17 700, 1998.

Sckopke, N., Paschmann, G., Brinca, A. L., Carlson, C. W., and Luehr, H. : Ion thermalization in quasi-perpendicular shocks involving reflected ions, J. Geophys. Res., 95, 6337-6352, 1990.

Song, P., Russell, C. T., and Thomsen, M. F.: Slow mode transition in the frontside magnetosheath, J. Geophys. Res., 97, 82958305, 1992.

Sonnerup, B. U. O.: The reconnecting magnetopause, in: Magnetospheric Physics, (Ed) McCormac, B. M., 23-33, D. Reidel, Norwell, Mass., 1974.

Sonnerup, B. U. O.: Theory of the low-latitude boundary layer, J. Geophys. Res., 85, 2017-2026, 1980.

Sonnerup, B. U. O. and Cahill, L. J.: Magnetopause structure and attitude from Explorer 12 observations, J. Geophys. Res., 72, 171-183, 1967.

Sonnerup, B. U. O and Siebert, K. D.: Theory of the low-latitude boundary layer and its coupling to the ionosphere: A tutorial review, in: Earth's Low-Latitude Boundary Layer, (Ed) Newell, P. T., and Onsager, T., Geophys. Monograph 133, AGU, 13-32, 2003.

Spreiter, J. R. and Alksne, A. Y.: Plasma flow around the magnetosphere, Rev. Geophys., 7, 11-50, 1969.

Spreiter, J. R., Summers, A. L., and Alksne, A. Y.: Hydromagnetic flow around the magnetosphere, Planet. Space Sci., 14, 223-253, 1966. 
Spreiter, J. R. and Stahara, S.: A new predictive model for determining solar wind-terrestrial planet interaction, J. Geophys. Res., 85, 6769-6777, 1980.

Terasawa, T., Kasaba, Y., Tsubouchi, K., Mukai, T., Saito, Y., Frank, L. A., et al.: Geotail observations of anonalously low density solar wind in the magnetosheath, Geophys. Res. Lett., 27, 3781-3784, 2000.

Treumann, R. A. and Baumjohann, W.: Advanced Space Plasma Physics, Imperial College Press, 1997.
Wang, Y. L., Raeder, J., Russell, C. T., Phan, T. D., and Manapat, M.: Plasma depletion layer: Event studies with a global model, J. Geophys. Res., doi:10.1029/2002JA009281, 108, 2003.

Zwan, B. J. and Wolf, R. A.: Depletion of the solar wind plasma near a planetary boundary, J. Geophys. Res., 81, 1636-1648, 1976. 